
Not for reproduction, distribution or commercial use.

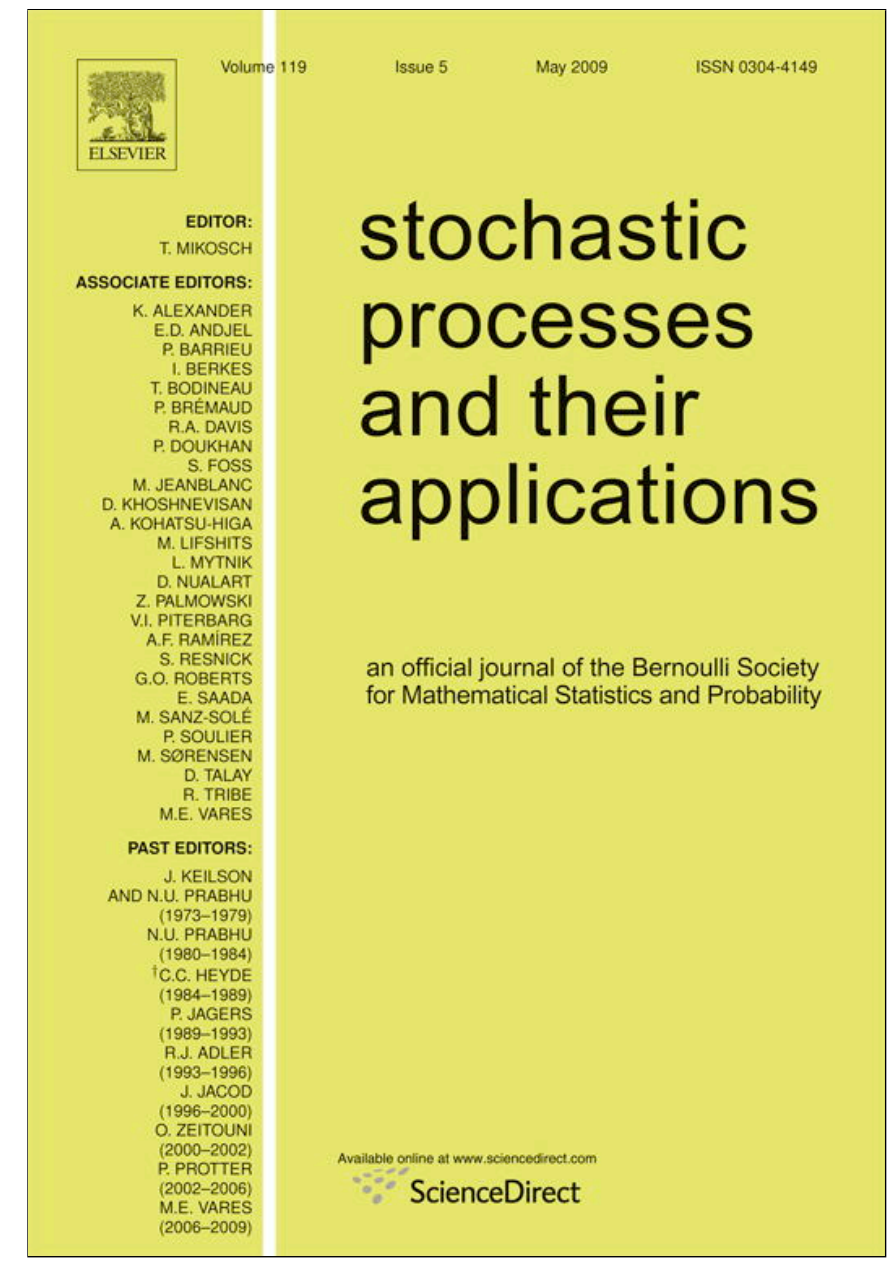

This article appeared in a journal published by Elsevier. The attached copy is furnished to the author for internal non-commercial research and education use, including for instruction at the authors institution and sharing with colleagues.

Other uses, including reproduction and distribution, or selling or licensing copies, or posting to personal, institutional or third party websites are prohibited.

In most cases authors are permitted to post their version of the article (e.g. in Word or Tex form) to their personal website or institutional repository. Authors requiring further information regarding Elsevier's archiving and manuscript policies are encouraged to visit:

http://www.elsevier.com/copyright 


\title{
Parametric estimation for partially hidden diffusion processes sampled at discrete times
}

\author{
Stefano Maria Iacus ${ }^{a}$, Masayuki Uchida ${ }^{b, *}$, Nakahiro Yoshida ${ }^{c, d}$ \\ ${ }^{a}$ Department of Economics, Business and Statistics, University of Milan, Via Conservatorio 7, 20122 Milan, Italy \\ ${ }^{\mathrm{b}}$ Graduate School of Engineering Science, Osaka University, Toyonaka, Osaka 560-8531, Japan \\ ${ }^{\mathrm{c}}$ Graduate School of Mathematical Sciences, University of Tokyo, 3-8-1 Komaba, Meguro-ku, Tokyo 153-8914, Japan \\ $\mathrm{d}$ Japan Science and Technology Agency (JST), Japan
}

Received 1 February 2008; received in revised form 28 July 2008; accepted 12 August 2008

Available online 19 August 2008

\begin{abstract}
For a one-dimensional diffusion process $X=\{X(t) ; 0 \leq t \leq T\}$, we suppose that $X(t)$ is hidden if it is below some fixed and known threshold $\tau$, but otherwise it is visible. This means a partially hidden diffusion process. The problem treated in this paper is the estimation of a finite-dimensional parameter in both drift and diffusion coefficients under a partially hidden diffusion process obtained by a discrete sampling scheme. It is assumed that the sampling occurs at regularly spaced time intervals of length $h_{n}$ such that $n h_{n}=T$. The asymptotic is when $h_{n} \rightarrow 0, T \rightarrow \infty$ and $n h_{n}^{2} \rightarrow 0$ as $n \rightarrow \infty$. Consistency and asymptotic normality for estimators of parameters in both drift and diffusion coefficients are proved.

(c) 2008 Elsevier B.V. All rights reserved.
\end{abstract}

MSC: primary 62F12; 62M05; secondary $60 \mathrm{~J} 60$

Keywords: Discrete observations; Partially observed systems; Diffusion processes

\section{Introduction}

We consider the estimation of the unknown parameter $\theta=\left(\theta_{1}, \theta_{2}\right)$ characterizing a onedimensional diffusion process defined by the stochastic differential equation

$$
\mathrm{d} X(t)=b\left(X(t), \theta_{2}\right) \mathrm{d} t+\sigma\left(X(t), \theta_{1}\right) \mathrm{d} W_{t}, \quad X(0)=x_{0}, \quad t \in[0, T],
$$

\footnotetext{
* Corresponding author. Tel.: +81 66850 6465; fax: +81 668506496 .

E-mail address: uchida@ sigmath.es.osaka-u.ac.jp (M. Uchida).
} 
where $W$ is a one-dimensional standard Brownian motion, $b$ and $\sigma$ are supposed to be regular enough to ensure the existence of a (strong) solution to the above stochastic differential equation. In the situation where discrete observations are $\mathbf{X}_{n}=\left\{X\left(t_{i}\right) ; i=0,1, \ldots, n\right\}$ with $t_{i}=i h_{n}$, $n h_{n}=T$, the estimation problem for the parameter $\theta$ has been considered by several authors, see $[2,11,12,16,17,9]$. In this paper, however, we generalize it to a different setup. We suppose that $X(t)$ is observable if $X(t)>\tau$ for some threshold $\tau$, and that $X(t)$ cannot be observed if $X(t) \leq \tau$. This means that the original process becomes a partially hidden diffusion process based on a threshold $\tau$, and the discretized trajectory $\mathbf{X}_{n}$ is also influenced by a threshold $\tau$. This type of observation naturally arises in the study of stochastic resonance and has been treated so far in the statistical context for the i.i.d. case in [4], for continuous time ergodic diffusion processes in [6] and for a class of continuous time mixing processes in [7]. In signal theory this corresponds to the problem of signal detection when the signal is so faint that it is not always receivable by some detector. This scheme of observation frequently appears in radio and $\mathrm{CCD}$ astronomy in the problem of identification of faint perturbed signals originated by astronomical sources (see e.g. [14]). A partially observed diffusion model also arises in the context of financial markets (see e.g. [18]) and in neuronal activation analysis (see e.g. [10]). In stochastic resonance context the original observation is altered by adding some noise with known structure to the channel in order to have full (but eventually quite noisy) observations, hence the problem is the one of determining the optimal level of noise. In the approach used in this paper, only the available observations are retained and used to estimate $\theta$. In this setup, we need to build a contrast function which is different from the one proposed in the literature of estimation for discretely observed diffusion processes cited above. Other different approaches based on particle filters (see e.g. [1]) and observation augmentation (see e.g. [13]) have been also recently proposed in the literature but our approach and asymptotic scheme adopted are substantially different from these references. Nevertheless, after some refinement it is still possible to prove consistency and asymptotic normality of the proposed estimators along the lines of e.g. [15,16,3, 9]. The organization of the paper is as follows. Section 2 introduces the model, the assumptions and two contrast functions. Section 3 contains the statement of the main result on consistency and asymptotic normality of estimators. Section 4 is devoted to the proofs of the results in Section 3.

\section{Model of observation and assumptions}

Let $X=\{X(t) ; 0 \leq t \leq T\}$ denote a diffusion process satisfying

$$
\mathrm{d} X(t)=b\left(X(t), \theta_{2}\right) \mathrm{d} t+\sigma\left(X(t), \theta_{1}\right) \mathrm{d} W_{t}, \quad X(0)=x_{0}, \quad t \in[0, T] .
$$

The parameter of our interest is $\theta=\left(\theta_{1}, \theta_{2}\right), \theta \in \Theta$ and $\Theta$ is a compact rectangle in $\mathbf{R}^{2}$. The true value is denoted by $\theta_{0}=\left(\theta_{1,0}, \theta_{2,0}\right)$ and it is assumed that $\theta_{0} \in \operatorname{Int}(\Theta)$. Let $X_{i}=X\left(t_{i}\right)$, $t_{i}=i h_{n}$ and $n h_{n}=T$. For $i=0,1, \ldots, n$, we assume that $X_{i}$ is observable if $X_{i}>\tau$ for some threshold $\tau$, and that $X_{i}$ is unobserved if $X_{i} \leq \tau$. The asymptotics will be investigated when $h_{n} \rightarrow 0, n h_{n} \rightarrow \infty$ and $n h_{n}^{2} \rightarrow 0$ as $n \rightarrow \infty$. In order to simplify the description, we use the following notation

$$
\sigma_{i}=\sigma\left(X_{i}, \theta_{1}\right), \quad b_{i}=b\left(X_{i}, \theta_{2}\right), \quad \Delta_{i} X=X_{i}-X_{i-1} .
$$

When the coefficients are evaluated at the true value of the parameter, we will write

$$
\sigma_{i}^{*}=\sigma\left(X_{i}, \theta_{1,0}\right), \quad b_{i}^{*}=b\left(X_{i}, \theta_{2,0}\right) .
$$


We further define $\partial_{\theta_{i}} f=\frac{\partial}{\partial \theta_{i}} f$. For any real sequence $u_{n} \in(0,1], R\left(u_{n}, x\right)$ represents a function such that

$$
\left|R\left(u_{n}, x\right)\right| \leq u_{n} C(1+|x|)^{C},
$$

where $C$ is a positive constant independent of $n$ and $x$ (and eventually $\theta$ when $x$ is $X(t)$ ). In the proof, $K$ and/or $C$ denote generic constants independent of $\theta, x$ and $n$.

\section{Assumptions}

A1 There exists $K>0$ such that for every $x, y \in \mathbf{R}$,

$$
\left|b\left(x, \theta_{2,0}\right)-b\left(y, \theta_{2,0}\right)\right|+\left|\sigma\left(x, \theta_{1,0}\right)-\sigma\left(y, \theta_{1,0}\right)\right| \leq K|x-y|,
$$

so that (1) has a unique solution for $\theta=\theta_{0}$.

A2 The process $X$ is stationary and ergodic for $\theta=\theta_{0}$ with its invariant measure denoted by $v_{\theta_{0}}$.

A3 For all $p \geq 0, \mathrm{E}\left[|X(0)|^{p}\right]<\infty$.

A4 $\inf _{x, \theta_{1}} \sigma^{2}\left(x, \theta_{1}\right)=K_{4}>0$.

A5 (Polynomial growth) The coefficients $b$ and $\sigma$ are continuously differentiable with respect to $x$ up to order 2 for all $\theta_{1}$ and $\theta_{2}$. These coefficients and their derivatives up to order 2 are of polynomial growth in $x$, uniformly in $\theta$.

A6 (Polynomial growth) The coefficients $b$ and $\sigma$ and all their $x$ derivatives up to order 2, are three times continuously differentiable with respect to $\theta$ for all $x$. Moreover, these $\theta$ derivatives are of polynomial growth in $x$ and uniformly on $\theta$.

A7 (Identifiability) $\sigma^{2}\left(x, \theta_{1}\right)=\sigma^{2}\left(x, \theta_{1,0}\right)$ for $v_{\theta_{0}}$ a.s. all $x \Rightarrow \theta_{1}=\theta_{1,0}$, $b\left(x, \theta_{2}\right)=b\left(x, \theta_{2,0}\right)$ for $v_{\theta_{0}}$ a.s. all $x \Rightarrow \theta_{2}=\theta_{2,0}$.

\section{The contrast function}

The main idea of this paper is to fix a new threshold $\tau^{\prime}(>\tau)$ as follows. We fix a number $\alpha \in(0,1 / 2)$ and take a sequence $\tau_{n}(>\tau)$ such that $h_{n}^{\alpha} /\left(\tau_{n}-\tau\right)=O(1)$; for example, $\tau_{n}=\tau+h_{n}^{\alpha}$. We use $\tau^{\prime}$ instead of $\tau_{n}$. Notice that $\tau^{\prime} \rightarrow \tau$ slowly. Thus, we introduce the following contrast functions

$$
\begin{aligned}
& g_{n}\left(\theta_{1}\right)=\sum_{i=1}^{n} g\left(i, i-1 ; \theta_{1}\right) \chi_{\left\{X_{i-1}>\tau^{\prime}, X_{i}>\tau\right\}}, \\
& \ell_{n}(\theta)=\sum_{i=1}^{n} \ell(i, i-1 ; \theta) \chi_{\left\{X_{i-1}>\tau^{\prime}, X_{i}>\tau\right\}},
\end{aligned}
$$

where $\chi$ is the indicator function and

$$
\begin{aligned}
& g\left(i, i-1 ; \theta_{1}\right)=\log \sigma_{i-1}^{2}+\frac{\left(\Delta_{i} X\right)^{2}}{\sigma_{i-1}^{2} h_{n}} \\
& \ell(i, i-1 ; \theta)=\log \sigma_{i-1}^{2}+\frac{\left(\Delta_{i} X-b_{i-1} h_{n}\right)^{2}}{\sigma_{i-1}^{2} h_{n}} .
\end{aligned}
$$

\section{Consistent and asymptotically normal estimators}

As in [16], we first estimate the parameter belonging to the diffusion coefficient, i.e. $\theta_{1}$, because, as usual, the estimator of $\theta_{1}$ has a faster rate of convergence than the one of $\theta_{2}$. Let $\hat{\theta}_{1, n}$ denote an estimator of $\theta_{1}$ satisfying 


$$
g_{n}\left(\hat{\theta}_{1, n}\right)=\inf _{\theta_{1}} g_{n}\left(\theta_{1}\right)
$$

The measurable selection theorem ensures the existence of such a measurable mapping.

Theorem 3.1. Under assumptions $\mathrm{A} 1-\mathrm{A} 7$, as $h_{n} \rightarrow 0$ and $n h_{n} \rightarrow \infty$,

$$
\hat{\theta}_{1, n} \stackrel{p}{\rightarrow} \theta_{1,0} .
$$

We consider an estimator $\hat{\theta}_{2, n}$ of $\theta_{2}$ that satisfies

$$
\ell_{n}\left(\hat{\theta}_{1, n}, \hat{\theta}_{2, n}\right)=\inf _{\theta_{2}} \ell_{n}\left(\hat{\theta}_{1, n}, \theta_{2}\right)
$$

Theorem 3.2. Under assumptions $\mathrm{A} 1-\mathrm{A} 7$, as $h_{n} \rightarrow 0$ and $n h_{n} \rightarrow \infty$,

$$
\hat{\theta}_{2, n} \stackrel{p}{\rightarrow} \theta_{2,0} .
$$

Let

$$
\Sigma=\left(\begin{array}{cc}
2 \int\left(\frac{\partial_{\theta_{1}} \sigma\left(x, \theta_{1,0}\right)}{\sigma\left(x, \theta_{1,0}\right)}\right)^{2} \chi_{\{x>\tau\}} \nu_{\theta_{0}}(\mathrm{~d} x) & 0 \\
0 & \int\left(\frac{\partial_{\theta_{2}} b\left(x, \theta_{2,0}\right)}{\sigma\left(x, \theta_{1,0}\right)}\right)^{2} \chi_{\{x>\tau\}} v_{\theta_{0}}(\mathrm{~d} x)
\end{array}\right) .
$$

The next theorem is the main result in this paper.

Theorem 3.3. Suppose that the assumptions A1-A7 are satisfied. If $\Sigma$ is non-singular, then as $h_{n} \rightarrow 0, n h_{n} \rightarrow \infty$ and $n h_{n}^{2} \rightarrow 0$,

$$
\left(\begin{array}{c}
\sqrt{n}\left(\hat{\theta}_{1, n}-\theta_{1,0}\right) \\
\sqrt{n h_{n}}\left(\hat{\theta}_{2, n}-\theta_{2,0}\right)
\end{array}\right) \stackrel{d}{\rightarrow} N\left(0, \Sigma^{-1}\right) .
$$

Remark 1. (i) As seen from the proof of (14), stationarity of the diffusion is used in order to show that $P\left(\tau^{\prime}<X_{i-1} \leq \tilde{\tau}\right)=o(1)$ and that $P\left(\tau^{\prime \prime}<X_{i-1} \leq \tau^{\prime}\right)=o(1)$, where $\tilde{\tau}=\tau^{\prime}+h_{n}^{\alpha}$ and $\tau^{\prime \prime}=\tau^{\prime}-h_{n}^{\alpha}$ for $\alpha \in(0,1 / 2)$. If we suppose A1 and A2 except for stationarity, the moment condition satisfying that $\sup _{t} \mathrm{E}\left[|X(t)|^{p}\right]<\infty$ for all $p \geq 0$, and A4-A7 together with regularity conditions for which the above estimates hold, Theorems 3.1-3.3 still hold true. (ii) It seems true that under some regularity conditions, Theorem 3.1 still holds even if $T\left(=n h_{n}\right)$ is fixed. For the case that $T$ is fixed, consistency and asymptotically mixed normality of the estimator will be a future work.

\section{Proofs}

Proof of Theorem 3.1. First, we will show that

$$
\sup _{\theta_{1}}\left|\frac{1}{n} g_{n}\left(\theta_{1}\right)-G\left(\theta_{1}\right)\right| \stackrel{p}{\rightarrow} 0
$$


where

$$
G\left(\theta_{1}\right)=\int_{\mathbf{R}}\left\{\log \sigma^{2}\left(x, \theta_{1}\right)+\frac{\sigma^{2}\left(x, \theta_{1,0}\right)}{\sigma^{2}\left(x, \theta_{1}\right)}\right\} \chi_{\{x>\tau\}} v_{\theta_{0}}(\mathrm{~d} x) .
$$

Noting that

$$
\chi_{\left\{X_{i-1}>\tau^{\prime}, X_{i}>\tau\right\}}-\chi_{\left\{X_{i-1}>\tau^{\prime}\right\}}=-\chi_{\left\{X_{i-1}>\tau^{\prime}, X_{i} \leq \tau\right\}},
$$

one has

$$
\begin{aligned}
\frac{1}{n} g_{n}\left(\theta_{1}\right)= & \frac{1}{n} \sum_{i=1}^{n} g\left(i, i-1 ; \theta_{1}\right) \chi_{\left\{X_{i-1}>\tau^{\prime}\right\}} \\
& -\frac{1}{n} \sum_{i=1}^{n} g\left(i, i-1 ; \theta_{1}\right) \chi_{\left\{X_{i-1}>\tau^{\prime}, X_{i} \leq \tau\right\}}
\end{aligned}
$$

In order to show the uniform convergence of (10) to zero, we consider the estimate that

$$
\begin{aligned}
& \mathrm{E}\left\{\sup _{\theta_{1}}\left|\frac{1}{n} \sum_{i=1}^{n} g\left(i, i-1 ; \theta_{1}\right) \chi_{\left\{X_{i-1}>\tau^{\prime}, X_{i} \leq \tau\right\}}\right|\right\} \\
& \quad \leq \frac{1}{n} \sum_{i=1}^{n}\left\|\sup _{\theta_{1}}\left|\log \sigma_{i-1}^{2}+\frac{\left(\Delta_{i} X\right)^{2}}{h_{n} \sigma_{i-1}^{2}}\right|\right\|_{p} P\left(X_{i-1}>\tau^{\prime}, X_{i} \leq \tau\right)^{\frac{1}{q}}
\end{aligned}
$$

for $1<p, q<\infty$ with $1 / p+1 / q=1$. Since A4 and A5 imply that

$$
\sup _{\theta_{1}}\left|\log \sigma_{i-1}^{2}\right| \leq \max \left(\left|\log \left(K_{4}\right)^{2}\right|, \sup _{\theta_{1}}\left|\sigma_{i-1}^{2}\right|\right) \leq K_{4}^{\prime}+C\left(1+\left|X_{i-1}\right|\right)^{C},
$$

it follows from A3 that

$$
\left\|\sup _{\theta_{1}}\left|\log \sigma_{i-1}^{2}\right|\right\|_{p}<\infty
$$

By A4 and the estimate that $\mathrm{E}\left|X_{i}-X_{i-1}\right|^{2 p} \leq C h_{n}^{p}$ for $p \geq 1$,

$$
\left\|\sup _{\theta_{1}} \frac{\left(\Delta_{i} X\right)^{2}}{h_{n} \sigma_{i-1}^{2}}\right\|_{p}^{p} \leq K\left\|\frac{\left(\Delta_{i} X\right)^{2}}{h_{n}}\right\|_{p}^{p}=O(1) .
$$

Moreover, for $k>0$,

$$
\begin{aligned}
\sup _{i} P\left(X_{i-1}>\tau^{\prime}, X_{i} \leq \tau\right) & \leq \sup _{i} P\left(\left|X_{i-1}-X_{i}\right| \geq \tau^{\prime}-\tau\right) \\
& \leq\left(\frac{1}{\tau^{\prime}-\tau}\right)^{k} \sup _{i} \mathrm{E}\left|X_{i-1}-X_{i}\right|^{k} \\
& \leq C\left(\frac{h_{n}^{\alpha}}{\tau^{\prime}-\tau}\right)^{k}\left(h_{n}^{1 / 2-\alpha}\right)^{k} \\
& =O\left(h_{n}^{(1 / 2-\alpha) k}\right) \rightarrow 0
\end{aligned}
$$


because $h_{n}^{\alpha} /\left(\tau^{\prime}-\tau\right)=O(1)$ for $\alpha \in(0,1 / 2)$. Thus, we obtain

$$
\sup _{\theta_{1}}\left|\frac{1}{n} \sum_{i=1}^{n} g(i, i-1 ; \theta) \chi_{\left\{X_{i-1}>\tau^{\prime}, X_{i} \leq \tau\right\}}\right|=o_{p}(1) .
$$

In order to prove the uniform convergence of (9) to $G$, it is enough to show that

$$
\frac{1}{n} \sum_{i=1}^{n} g\left(i, i-1 ; \theta_{1}\right) \chi_{\left\{X_{i-1}>\tau^{\prime}\right\}} \stackrel{p}{\rightarrow} G\left(\theta_{1}\right)
$$

for each $\theta_{1}$, and

$$
\sup _{n} E\left[\sup _{\theta_{1}}\left|\frac{1}{n} \sum_{i=1}^{n} \partial_{\theta_{1}} g\left(i, i-1 ; \theta_{1}\right)\right|\right]<\infty .
$$

For details, see the proof of Theorem 4.1 in [15]. As in the proof of the uniform convergence of (10), we can obtain (13). For the proof of (12), we will prove

$$
\begin{aligned}
& \frac{1}{n} \sum_{i=1}^{n} \log \sigma_{i-1}^{2} \chi_{\left\{X_{i-1}>\tau^{\prime}\right\}} \stackrel{p}{\rightarrow} \int_{\mathbf{R}} \log \sigma^{2}(x, \theta) \chi_{\{x>\tau\}} v_{\theta_{0}}(\mathrm{~d} x), \\
& \frac{1}{n h_{n}} \sum_{i=1}^{n} \frac{\left(\Delta_{i} X\right)^{2}}{\sigma_{i-1}^{2}} \chi_{\left\{X_{i-1}>\tau^{\prime}\right\}} \stackrel{p}{\rightarrow} \int_{\mathbf{R}} \frac{\sigma^{2}\left(x, \theta_{1,0}\right)}{\sigma^{2}\left(x, \theta_{1}\right)} \chi_{\{x>\tau\}} v_{\theta_{0}}(\mathrm{~d} x) .
\end{aligned}
$$

For the proof of (14), we set $I_{i}=\int_{t_{i-1}}^{t_{i}} \log \sigma_{i-1}^{2} \chi_{\left\{X_{i-1}>\tau^{\prime}\right\}} \mathrm{d} s$ for $i=1, \ldots, n$. Note that

$$
\chi_{\left\{X_{i-1}>\tau^{\prime}\right\}} \geq \chi_{\left\{\inf _{s \in\left(t_{i-1}, t_{i}\right]} X(s)>\tau\right\}}-\chi_{\left\{\tau<\inf _{s \in\left(t_{i-1}, t_{i}\right]} X(s) \leq \tau^{\prime}\right\}}
$$

We first estimate $I_{i}$ for the case that $\log \sigma_{i-1}^{2} \geq 0$. Let $J_{i}=\chi_{\left\{\log \sigma_{i-1}^{2} \geq 0\right\}}$ for $i=1, \ldots, n$.

$$
\begin{aligned}
I_{i} J_{i}= & J_{i} \int_{t_{i-1}}^{t_{i}} \log \sigma_{i-1}^{2} \chi_{\left\{X_{i-1}>\tau^{\prime}\right\}}^{2} \mathrm{~d} s \\
\geq & J_{i} \int_{t_{i-1}}^{t_{i}} \log \sigma_{i-1}^{2} \chi_{\left\{X_{i-1}>\tau^{\prime}\right\}}\left[\chi_{\left\{\inf _{s \in\left(t_{i-1}, t_{i}\right]} X(s)>\tau\right\}}-\chi_{\left\{\tau<\inf _{\left.s \in t_{i-1}, t_{i}\right]} X(s) \leq \tau^{\prime}\right\}}\right] \mathrm{d} s \\
\geq & -J_{i} \int_{t_{i-1}}^{t_{i}} \log \sigma_{i-1}^{2} \chi_{\left\{X_{i-1}>\tau^{\prime}\right\}} \chi_{\left\{\tau<\inf _{s \in\left(t_{i-1}, t_{i}\right]} X(s) \leq \tau^{\prime}\right\}} \mathrm{d} s \\
& +J_{i} \int_{t_{i-1}}^{t_{i}} \log \sigma_{i-1}^{2} \chi_{\left\{X_{i-1}>\tau^{\prime}\right\}} \chi_{\left\{\inf _{s \in\left(t_{i-1}, t_{i}\right]} X(s)>\tau\right\}} \chi_{\{X(s)>\tau\}} \mathrm{d} s \\
= & -J_{i} \int_{t_{i-1}}^{t_{i}} \log \sigma_{i-1}^{2} \chi_{\left\{X_{i-1}>\tau^{\prime}\right\}} \chi_{\left\{\tau<\inf _{s \in\left(t_{i-1}, t_{i}\right]} X(s) \leq \tau^{\prime}\right\}} \mathrm{d} s \\
& +J_{i} \int_{t_{i-1}}^{t_{i}} \log \sigma_{i-1}^{2} \chi_{\left\{X_{i-1}>\tau^{\prime}\right\}}\left[\chi_{\left\{\inf _{s \in\left(t_{i-1}, t_{i}\right]} X(s)>\tau\right\}}-1\right] \chi_{\{X(s)>\tau\}} \mathrm{d} s \\
& +J_{i} \int_{t_{i-1}}^{t_{i}} \log \sigma_{i-1}^{2}\left[\chi_{\left\{X_{i-1}>\tau^{\prime}\right\}}-1\right] \chi_{\{X(s)>\tau\}} \mathrm{d} s+J_{i} \int_{t_{i-1}}^{t_{i}} \log \sigma_{i-1}^{2} \chi_{\{X(s)>\tau\}} \mathrm{d} s .
\end{aligned}
$$


Hence,

$$
J_{i}\left(I_{i}-\int_{t_{i-1}}^{t_{i}} \log \sigma^{2}\left(X(s), \theta_{1}\right) \chi\{X(s)>\tau\} \mathrm{d} s\right) \geq J_{i} \Xi_{i}^{(1)},
$$

where

$$
\begin{aligned}
\Xi_{i}^{(1)}= & -\int_{t_{i-1}}^{t_{i}} \log \sigma_{i-1}^{2} \chi_{\left\{X_{i-1}>\tau^{\prime}\right\}} \chi_{\left\{\tau<\inf _{s \in\left(t_{i-1}, t_{i}\right]} X(s) \leq \tau^{\prime}\right\}} \mathrm{d} s \\
& -\int_{t_{i-1}}^{t_{i}} \log \sigma_{i-1}^{2} \chi_{\left\{X_{i-1}>\tau^{\prime}\right\}} \chi_{\left\{\inf _{s \in\left(t_{i-1}, t_{i}\right]} X(s) \leq \tau\right\}} \chi_{\{X(s)>\tau\}} \mathrm{d} s \\
& -\int_{t_{i-1}}^{t_{i}} \log \sigma_{i-1}^{2} \chi_{\left\{X_{i-1} \leq \tau^{\prime}\right\}} \chi_{\{X(s)>\tau\}} \mathrm{d} s \\
& +\int_{t_{i-1}}^{t_{i}}\left\{\log \sigma_{i-1}^{2}-\log \sigma^{2}\left(X(s), \theta_{1}\right)\right\} \chi_{\{X(s)>\tau\}} \mathrm{d} s .
\end{aligned}
$$

Next, noting that

$$
\begin{aligned}
I_{i} J_{i}= & J_{i} \int_{t_{i-1}}^{t_{i}} \log \sigma_{i-1}^{2} \chi_{\left\{X_{i-1}>\tau^{\prime}\right\}} \chi_{\left\{\inf _{s \in\left(t_{i-1}, t_{i}\right]} X(s)>\tau\right\}} \mathrm{d} s \\
& +J_{i} \int_{t_{i-1}}^{t_{i}} \log \sigma_{i-1}^{2} \chi_{\left\{X_{i-1}>\tau^{\prime}\right\}} \chi_{\left\{\inf _{\left.s \in t_{i-1}, t_{i}\right]} X(s) \leq \tau\right\}} \mathrm{d} s \\
\leq & J_{i} \int_{t_{i-1}}^{t_{i}} \log \sigma_{i-1}^{2} \chi_{\{X(s)>\tau\}} \mathrm{d} s+J_{i} \int_{t_{i-1}}^{t_{i}} \log \sigma_{i-1}^{2} \chi_{\left\{X_{i-1}>\tau^{\prime}\right\}} \chi_{\left\{\inf _{\left.s \in t_{i-1}, t_{i}\right]} X(s) \leq \tau\right\}} \mathrm{d} s,
\end{aligned}
$$

we obtain that

$$
J_{i}\left(I_{i}-\int_{t_{i-1}}^{t_{i}} \log \sigma^{2}\left(X(s), \theta_{1}\right) \chi_{\{X(s)>\tau\}} \mathrm{d} s\right) \leq J_{i} \Xi_{i}^{(2)}
$$

where

$$
\begin{aligned}
\Xi_{i}^{(2)}= & \int_{t_{i-1}}^{t_{i}}\left\{\log \sigma_{i-1}^{2}-\log \sigma^{2}\left(X(s), \theta_{1}\right)\right\} \chi_{\{X(s)>\tau\}} \mathrm{d} s \\
& +\int_{t_{i-1}}^{t_{i}} \log \sigma_{i-1}^{2} \chi_{\left\{X_{i-1}>\tau^{\prime}\right\}} \chi_{\left\{\inf _{\left.s \in t_{i-1}, t_{i}\right]} X(s) \leq \tau\right\}} \mathrm{d} s .
\end{aligned}
$$

It follows from (17) and (22) that

$$
\left|J_{i}\left(I_{i}-\int_{t_{i-1}}^{t_{i}} \log \sigma^{2}\left(X(s), \theta_{1}\right) \chi_{\{X(s)>\tau\}} \mathrm{d} s\right)\right| \leq \max \left\{\left|\Xi_{i}^{(1)}\right|,\left|\Xi_{i}^{(2)}\right|\right\} .
$$

For the estimate of (18), we set $\tilde{\tau}=\tau^{\prime}+h_{n}^{\alpha}$, where $\alpha \in(0,1 / 2)$.

$$
\begin{aligned}
& E\left[\left|\int_{t_{i-1}}^{t_{i}} \log \sigma_{i-1}^{2} \chi_{\left\{X_{i-1}>\tau^{\prime}\right\}} \chi_{\left\{\tau<\inf _{s \in\left(t_{i-1}, t_{i}\right]} X(s) \leq \tau^{\prime}\right\}} \mathrm{d} s\right|\right] \\
& \quad \leq E\left[\int_{t_{i-1}}^{t_{i}}\left|\log \sigma_{i-1}^{2}\right|\left\{\chi_{\left\{X_{i-1}>\tau^{\prime}\right\}}-\chi_{\left\{X_{i-1}>\tilde{\tau}\right\}}\right\} \chi_{\left\{\tau<\inf _{s \in\left(t_{i-1}, t_{i}\right]} X(s) \leq \tau^{\prime}\right\}} \mathrm{d} s\right]
\end{aligned}
$$




$$
\begin{aligned}
& +E\left[\int_{t_{i-1}}^{t_{i}}\left|\log \sigma_{i-1}^{2}\right| \chi_{\left\{X_{i-1}>\tilde{\tau}\right\}} \chi_{\left\{\tau<\inf _{\left.s \in t_{i-1}, t_{i}\right]} X(s) \leq \tau^{\prime}\right\}} \mathrm{d} s\right] \\
\leq & E\left[\int_{t_{i-1}}^{t_{i}}\left|\log \sigma_{i-1}^{2}\right| \chi_{\left\{\tau^{\prime}<X_{i-1} \leq \tilde{\tau}\right\}} \mathrm{d} s\right] \\
& +E\left[\int_{t_{i-1}}^{t_{i}}\left|\log \sigma_{i-1}^{2}\right| \chi_{\left\{X_{i-1}>\tilde{\tau}\right\}} \chi_{\left\{\inf _{\left.s \in t_{i-1}, t_{i}\right]} X(s) \leq \tau^{\prime}\right\}} \mathrm{d} s\right] \\
\leq & h_{n} C\left[P\left(\tau^{\prime}<X_{i-1} \leq \tilde{\tau}\right)^{1 / 2}+P\left(\sup _{s \in\left(t_{i-1}, t_{i}\right]}\left|X_{i-1}-X(s)\right|>h_{n}^{\alpha}\right)^{1 / 2}\right]=o\left(h_{n}\right) .
\end{aligned}
$$

Concerning the estimate of (19),

$$
\begin{gathered}
E\left[\left|\int_{t_{i-1}}^{t_{i}} \log \sigma_{i-1}^{2} \chi_{\left\{X_{i-1}>\tau^{\prime}\right\}} \chi_{\left\{\inf _{s \in\left(t_{i-1}, t_{i}\right]} X(s) \leq \tau\right\}} \chi_{\{X(s)>\tau\}} \mathrm{d} s\right|\right] \\
\quad \leq h_{n} C P\left(\sup _{s \in\left(t_{i-1}, t_{i}\right]}\left|X_{i-1}-X(s)\right|>h_{n}^{\alpha}\right)^{1 / 2}=o\left(h_{n}\right) .
\end{gathered}
$$

In order to estimate (20), we set $\tau^{\prime \prime}=\tau-h_{n}^{\alpha}$, where $\alpha \in(0,1 / 2)$.

$$
\begin{aligned}
E & {\left[\left|\int_{t_{i-1}}^{t_{i}} \log \sigma_{i-1}^{2} \chi_{\left\{X_{i-1} \leq \tau^{\prime}\right\}} \chi_{\{X(s)>\tau\}} \mathrm{d} s\right|\right] } \\
\leq & E\left[\int_{t_{i-1}}^{t_{i}}\left|\log \sigma_{i-1}^{2}\right|\left\{\chi_{\left\{X_{i-1} \leq \tau^{\prime}\right\}}-\chi_{\left\{\tau^{\prime \prime}<X_{i-1} \leq \tau^{\prime}\right\}}\right\} \chi_{\{X(s)>\tau\}} \mathrm{d} s\right] \\
& +E\left[\int_{t_{i-1}}^{t_{i}}\left|\log \sigma_{i-1}^{2}\right| \chi_{\left\{\tau^{\prime \prime}<X_{i-1} \leq \tau^{\prime}\right\}} \chi_{\{X(s)>\tau\}} \mathrm{d} s\right] \\
\leq & E\left[\int_{t_{i-1}}^{t_{i}}\left|\log \sigma_{i-1}^{2}\right| \chi_{\left\{X_{i-1} \leq \tau^{\prime \prime}\right\}} \chi_{\{X(s)>\tau\}} \mathrm{d} s\right]+E\left[\int_{t_{i-1}}^{t_{i}}\left|\log \sigma_{i-1}^{2}\right| \chi_{\left\{\tau^{\prime \prime}<X_{i-1} \leq \tau^{\prime}\right\}} \mathrm{d} s\right] \\
\leq & h_{n} C\left[P\left(\sup _{s \in\left(t_{i-1}, t_{i}\right]}\left|X(s)-X_{i-1}\right|>h_{n}^{\alpha}\right)^{1 / 2}+P\left(\tau^{\prime \prime}<X_{i-1} \leq \tau^{\prime}\right)^{1 / 2}\right]=o\left(h_{n}\right) .
\end{aligned}
$$

As for the estimate of (21),

$$
E\left[\left|\int_{t_{i-1}}^{t_{i}}\left\{\log \sigma_{i-1}^{2}-\log \sigma^{2}\left(X(s), \theta_{1}\right)\right\} \mathrm{d} s\right|\right] \leq C h_{n}^{3 / 2}=o\left(h_{n}\right) .
$$

Thus, we obtain

$$
E\left[\left|\Xi_{i}^{(1)}\right|\right]=o\left(h_{n}\right)
$$

Moreover,

$$
\begin{aligned}
E\left[\left|\Xi_{i}^{(2)}\right|\right] \leq & E\left[\left|\int_{t_{i-1}}^{t_{i}}\left\{\log \sigma_{i-1}^{2}-\log \sigma^{2}\left(X(s), \theta_{1}\right)\right\} \chi_{\{X(s)>\tau\}} \mathrm{d} s\right|\right] \\
& +E\left[\left|\int_{t_{i-1}}^{t_{i}} \log \sigma_{i-1}^{2} \chi_{\left\{X_{i-1}>\tau^{\prime}\right\}} \chi_{\left\{\inf _{s \in\left(t_{i-1}, t_{i}\right]} X(s) \leq \tau\right\}} \mathrm{d} s\right|\right] \\
= & o\left(h_{n}\right) .
\end{aligned}
$$


It follows from (23) and (24) that

$$
E\left[\left|J_{i} \int_{t_{i-1}}^{t_{i}}\left\{\log \sigma_{i-1}^{2} \chi_{\left\{X_{i-1}>\tau^{\prime}\right\}}-\log \sigma^{2}\left(X(s), \theta_{1}\right) \chi_{\{X(s)>\tau\}}\right\} \mathrm{d} s\right|\right]=o\left(h_{n}\right) .
$$

For the case that $\log \sigma_{i-1}^{2}<0$, set $\tilde{J}_{i}=\chi_{\left\{\log \sigma_{i-1}^{2}<0\right\}}$ for $i=1, \ldots, n$. In a similar way as the upper bound of $I_{i} J_{i}$ together with (16),

$$
\begin{aligned}
I_{i} \tilde{J}_{i}= & -\tilde{J}_{i} \int_{t_{i-1}}^{t_{i}}\left(-\log \sigma_{i-1}^{2}\right) \chi_{\left\{X_{i-1}>\tau^{\prime}\right\}} \mathrm{d} s=-\tilde{J}_{i} \int_{t_{i-1}}^{t_{i}}\left(-\log \sigma_{i-1}^{2}\right) \chi_{\left\{X_{i-1}>\tau^{\prime}\right\}}^{2} \mathrm{~d} s \\
\leq & -\tilde{J}_{i} \int_{t_{i-1}}^{t_{i}}\left(-\log \sigma_{i-1}^{2}\right) \chi_{\left\{X_{i-1}>\tau^{\prime}\right\}}\left[\chi_{\left\{\inf _{s \in\left(t_{i-1}, t_{i}\right]} X(s)>\tau\right\}}-\chi_{\left.\left\{\tau<\inf _{\left.s \in t_{i-1}, t_{i}\right]} X(s) \leq \tau^{\prime}\right\}\right] \mathrm{d} s}\right. \\
\leq & \tilde{J}_{i} \int_{t_{i-1}}^{t_{i}}\left(-\log \sigma_{i-1}^{2}\right) \chi_{\left\{X_{i-1}>\tau^{\prime}\right\}} \chi_{\left\{\tau<\inf _{s \in\left(t_{i-1}, t_{i}\right]} X(s) \leq \tau^{\prime}\right\}} \mathrm{d} s \\
& -\tilde{J}_{i} \int_{t_{i-1}}^{t_{i}}\left(-\log \sigma_{i-1}^{2}\right) \chi_{\left\{X_{i-1}>\tau^{\prime}\right\}} \chi_{\left\{\inf _{s \in\left(t_{i-1}, t_{i}\right]} X(s)>\tau\right\}} \chi_{\{X(s)>\tau\}} \mathrm{d} s \\
= & \tilde{J}_{i} \int_{t_{i-1}}^{t_{i}}\left(-\log \sigma_{i-1}^{2}\right) \chi_{\left\{X_{i-1}>\tau^{\prime}\right\}} \chi_{\left\{\tau<\inf _{s \in\left(t_{i-1}, t_{i}\right]} X(s) \leq \tau^{\prime}\right\}} \mathrm{d} s \\
& -\tilde{J}_{i} \int_{t_{i-1}}^{t_{i}}\left(-\log \sigma_{i-1}^{2}\right) \chi_{\left\{X_{i-1}>\tau^{\prime}\right\}}\left[\chi_{\left\{\inf _{s \in\left(t_{i-1}, t_{i}\right]} X(s)>\tau\right\}}-1\right] \chi_{\{X(s)>\tau\}} \mathrm{d} s \\
& -\tilde{J}_{i} \int_{t_{i-1}}^{t_{i}}\left(-\log \sigma_{i-1}^{2}\right)\left[\chi_{\left\{X_{i-1}>\tau^{\prime}\right\}}-1\right] \chi_{\{X(s)>\tau\}} \mathrm{d} s \\
& -\tilde{J}_{i} \int_{t_{i-1}}^{t_{i}}\left(-\log \sigma_{i-1}^{2}+\log \sigma^{2}\left(X(s), \theta_{1}\right)\right) \chi_{\{X(s)>\tau\}} \mathrm{d} s \\
& -\tilde{J}_{i} \int_{t_{i-1}}^{t_{i}}\left(-\log \sigma^{2}\left(X(s), \theta_{1}\right)\right) \chi_{\{X(s)>\tau\}} \mathrm{d} s .
\end{aligned}
$$

Therefore,

$$
\tilde{J}_{i}\left(I_{i}-\int_{t_{i-1}}^{t_{i}} \log \sigma^{2}\left(X(s), \theta_{1}\right) \chi\{X(s)>\tau\} \mathrm{d} s\right) \leq \tilde{J}_{i} \Xi_{i}^{(1)} .
$$

Moreover, since

$$
\begin{aligned}
I_{i} \tilde{J}_{i}= & -\tilde{J}_{i} \int_{t_{i-1}}^{t_{i}}\left(-\log \sigma_{i-1}^{2}\right) \chi_{\left\{X_{i-1}>\tau^{\prime}\right\}} \chi_{\left\{\inf _{\left.s \in t_{i-1}, t_{i}\right]} X(s)>\tau\right\}} \mathrm{d} s \\
& -\tilde{J}_{i} \int_{t_{i-1}}^{t_{i}}\left(-\log \sigma_{i-1}^{2}\right) \chi_{\left\{X_{i-1}>\tau^{\prime}\right\}} \chi_{\left\{\inf _{s \in\left(t_{i-1}, t_{i}\right]} X(s) \leq \tau\right\}} \mathrm{d} s \\
\geq & -\tilde{J}_{i} \int_{t_{i-1}}^{t_{i}}\left(-\log \sigma_{i-1}^{2}+\log \sigma^{2}\left(X(s), \theta_{1}\right)\right) \chi_{\{X(s)>\tau\}} \mathrm{d} s \\
& -\tilde{J}_{i} \int_{t_{i-1}}^{t_{i}}\left(-\log \sigma^{2}\left(X(s), \theta_{1}\right)\right) \chi_{\{X(s)>\tau\}} \mathrm{d} s \\
& -\tilde{J}_{i} \int_{t_{i-1}}^{t_{i}}\left(-\log \sigma_{i-1}^{2}\right) \chi_{\left\{X_{i-1}>\tau^{\prime}\right\}} \chi_{\left\{\inf _{s \in\left(t_{i-1}, t_{i}\right]} X(s) \leq \tau\right\}} \mathrm{d} s,
\end{aligned}
$$


one has that

$$
\tilde{J}_{i}\left(I_{i}-\int_{t_{i-1}}^{t_{i}} \log \sigma^{2}\left(X(s), \theta_{1}\right) \chi_{\{X(s)>\tau\}} \mathrm{d} s\right) \geq \tilde{J}_{i} \Xi_{i}^{(2)} .
$$

It follows from (26) and (27) that

$$
\left|\tilde{J}_{i}\left(I_{i}-\int_{t_{i-1}}^{t_{i}} \log \sigma^{2}\left(X(s), \theta_{1}\right) \chi_{\{X(s)>\tau\}} \mathrm{d} s\right)\right| \leq \max \left\{\left|\Xi_{i}^{(1)}\right|,\left|\Xi_{i}^{(2)}\right|\right\} .
$$

By (23) and (24), one has that

$$
E\left[\left|\left(1-J_{i}\right) \int_{t_{i-1}}^{t_{i}}\left\{\log \sigma_{i-1}^{2} \chi_{\left\{X_{i-1}>\tau^{\prime}\right\}}-\log \sigma^{2}\left(X(s), \theta_{1}\right) \chi_{\{X(s)>\tau\}}\right\} \mathrm{d} s\right|\right]=o\left(h_{n}\right) .
$$

Therefore, by (25) and (28),

$$
E\left[\left|\int_{t_{i-1}}^{t_{i}}\left\{\log \sigma_{i-1}^{2} \chi_{\left\{X_{i-1}>\tau^{\prime}\right\}}-\log \sigma^{2}\left(X(s), \theta_{1}\right) \chi_{\{X(s)>\tau\}}\right\} \mathrm{d} s\right|\right]=o\left(h_{n}\right)
$$

and consequently,

$$
\left|\frac{1}{n h_{n}} \sum_{i=1}^{n} \int_{t_{i-1}}^{t_{i}}\left\{\log \sigma_{i-1}^{2} \chi_{\left\{X_{i-1}>\tau^{\prime}\right\}}-\log \sigma^{2}\left(X(s), \theta_{1}\right) \chi_{\{X(s)>\tau\}}\right\} \mathrm{d} s\right|=o_{p}(1) .
$$

Moreover, by the ergodic theorem,

$$
\frac{1}{n h_{n}} \int_{0}^{n h_{n}} \log \sigma^{2}\left(X(s), \theta_{1}\right) \chi_{\{X(s)>\tau\}} \mathrm{d} s \stackrel{p}{\rightarrow} \int_{\mathbf{R}} \log \sigma^{2}\left(x, \theta_{1}\right) \chi_{\{x>\tau\}} v_{\theta_{0}}(\mathrm{~d} x),
$$

which completes the proof of (14). For the proof of (15), we set

$$
\Xi_{i}=\frac{1}{n h_{n}} \frac{\left(\Delta_{i} X\right)^{2}}{\sigma_{i-1}^{2}} \chi_{\left\{X_{i-1}>\tau^{\prime}\right\}}
$$

By Lemma 9 of [3], it is enough to show that

$$
\begin{aligned}
& \sum_{i=1}^{n} \mathrm{E}_{\theta_{0}}\left\{\Xi_{i} \mid \mathcal{F}_{i-1}\right\} \stackrel{p}{\rightarrow} \int_{\mathbf{R}} \frac{\sigma^{2}\left(x, \theta_{1,0}\right)}{\sigma^{2}\left(x, \theta_{1}\right)} \chi_{\{x>\tau\}} v_{\theta_{0}}(\mathrm{~d} x), \\
& \sum_{i=1}^{n} \mathrm{E}_{\theta_{0}}\left\{\left(\Xi_{i}\right)^{2} \mid \mathcal{F}_{i-1}\right\} \stackrel{p}{\rightarrow} 0,
\end{aligned}
$$

where $\mathcal{F}_{i-1}$ denotes the history up to the time $t_{i-1}$. In order to evaluate $\mathrm{E}_{\theta_{0}}\left\{\left(\Delta_{i} X\right)^{2} \mid \mathcal{F}_{i-1}\right\}$, we can use a well-known Itô-Taylor expansion:

$$
\begin{aligned}
\mathrm{E}_{\theta_{0}}\left(\phi\left(X_{i}, X_{i-1}\right) \mid \mathcal{F}_{i-1}\right) \\
=\phi\left(X_{i-1}, X_{i-1}\right)+h_{n} L_{\theta_{0}} \phi\left(X_{i-1}, X_{i-1}\right)+\frac{1}{2} h_{n}^{2} L_{\theta_{0}}^{2} \phi\left(X_{i-1}, X_{i-1}\right) \\
\quad+\int_{t_{i-1}}^{t_{i}} \int_{t_{i-1}}^{t} E_{\theta_{0}}\left\{L_{\theta_{0}}^{2} \phi\left(X(s), X_{i-1}\right)-L_{\theta_{0}}^{2} \phi\left(X_{i-1}, X_{i-1}\right) \mid \mathcal{F}_{i-1}\right\} \mathrm{d} s \mathrm{~d} t
\end{aligned}
$$


for appropriate functions $\phi(x, y)$, where $L_{\theta} \phi(x, y)=\frac{1}{2} \sigma^{2}\left(x, \theta_{1}\right) \frac{\partial^{2}}{\partial x^{2}} \phi(x, y)+b\left(x, \theta_{2}\right) \frac{\partial}{\partial x} \phi$ $(x, y)$. Hence

$$
\mathrm{E}_{\theta_{0}}\left\{\left(\Delta_{i} X\right)^{2} \mid \mathcal{F}_{i-1}\right\}=h_{n} \sigma_{i-1}^{* 2}+R\left(h_{n}^{2}, X_{i-1}\right),
$$

where $R(\cdot, \cdot)$ is defined in (2). Thus, as in the proof of (14),

$$
\begin{aligned}
\sum_{i=1}^{n} E_{\theta_{0}}\left\{\Xi_{i} \mid \mathcal{F}_{i-1}\right\} & =\frac{1}{n} \sum_{i=1}^{n} \frac{\left(\sigma_{i-1}^{*}\right)^{2}}{\sigma_{i-1}^{2}} \chi_{\left\{X_{i-1}>\tau^{\prime}\right\}}+\frac{h_{n}}{n} \sum_{i=1}^{n} R\left(1, X_{i-1}\right) \\
& \stackrel{p}{\rightarrow} \int_{\mathbf{R}} \frac{\sigma^{2}\left(x, \theta_{1,0}\right)}{\sigma^{2}\left(x, \theta_{1}\right)} \chi_{\{x>\tau\}} v_{\theta_{0}}(\mathrm{~d} x)
\end{aligned}
$$

and in a similar way, we can show (31). This completes the proof of (7).

Next, we see that $G$ attains its minimum only at $\theta_{1,0}$ by noting that

$$
\frac{\mathrm{d}}{\mathrm{d} x}\left(\log x+\frac{a}{x}\right)=\frac{1}{x}-\frac{a}{x^{2}}=\frac{x-a}{x^{2}} .
$$

Hence, for any $\epsilon>0, \inf _{\theta_{1}:\left|\theta_{1}-\theta_{1,0}\right| \geq \epsilon} G\left(\theta_{1}\right)>G\left(\theta_{1,0}\right)$. This implies that if $\left|\theta_{1}-\theta_{1,0}\right| \geq \epsilon$, then $G\left(\theta_{1}\right)>G\left(\theta_{1,0}\right)+\eta$ for some $\eta>0$. Therefore,

$$
\begin{aligned}
P\left(\left|\hat{\theta}_{1, n}-\theta_{1,0}\right| \geq \epsilon\right) \leq & P\left(G\left(\hat{\theta}_{1, n}\right)>G\left(\theta_{1,0}\right)+\eta\right) \\
\leq & 2 P\left(\sup _{\theta_{1}}\left|\frac{1}{n} g_{n}\left(\theta_{1}\right)-G\left(\theta_{1}\right)\right|>\eta / 3\right) \\
& +P\left(\frac{1}{n} g_{n}\left(\hat{\theta}_{1, n}\right)-\frac{1}{n} g_{n}\left(\theta_{1,0}\right)>\eta / 3\right) .
\end{aligned}
$$

By using (7), the probability of (33) converges to 0. Furthermore, it follows from (5) that

$$
P\left(\frac{1}{n} g_{n}\left(\hat{\theta}_{1, n}\right)-\frac{1}{n} g_{n}\left(\theta_{1,0}\right)>\eta / 3\right) \leq P\left(\frac{1}{n} g_{n}\left(\hat{\theta}_{1, n}\right)>\frac{1}{n} g_{n}\left(\theta_{1,0}\right)\right) \rightarrow 0 \text {. }
$$

This competes the proof.

Proof of Theorem 3.2. We need to prove that

$$
\sup _{\theta_{2}}\left|\frac{1}{n h_{n}}\left(\ell_{n}\left(\hat{\theta}_{1, n}, \theta_{2}\right)-\ell_{n}\left(\hat{\theta}_{1, n}, \theta_{2,0}\right)\right)-L\left(\theta_{2}\right)\right| \stackrel{p}{\rightarrow} 0,
$$

where

$$
L\left(\theta_{2}\right)=\int_{\mathbf{R}}\left(\frac{b\left(x, \theta_{2}\right)-b\left(x, \theta_{2,0}\right)}{\sigma\left(x, \theta_{1,0}\right)}\right)^{2} \chi_{\{x>\tau\}} v_{\theta_{0}}(\mathrm{~d} x) .
$$

An easy computation together with (8) yields that

$$
\frac{1}{n h_{n}}\left(\ell_{n}\left(\hat{\theta}_{1, n}, \theta_{2}\right)-\ell_{n}\left(\hat{\theta}_{1, n}, \theta_{2,0}\right)\right)=\psi_{1, n}\left(\theta_{2}\right)+\psi_{2, n}\left(\theta_{2}\right)+\psi_{3, n}\left(\theta_{2}\right)+R_{n}\left(\theta_{2}\right),
$$

where $\hat{\sigma}_{i}=\sigma\left(X_{i}, \hat{\theta}_{1, n}\right)$,

$$
\psi_{1, n}\left(\theta_{2}\right)=-\frac{2}{n h_{n}} \sum_{i=1}^{n} \frac{\left(b_{i-1}-b_{i-1}^{*}\right) \int_{t_{i-1}}^{t_{i}} \sigma\left(X(s), \theta_{1,0}\right) \mathrm{d} W_{s}}{\hat{\sigma}_{i-1}^{2}} \chi_{\left\{X_{i-1}>\tau^{\prime}\right\}}
$$




$$
\begin{aligned}
& \psi_{2, n}\left(\theta_{2}\right)=-\frac{2}{n h_{n}} \sum_{i=1}^{n} \frac{\left(b_{i-1}-b_{i-1}^{*}\right) \int_{t_{i-1}}^{t_{i}} b\left(X(s), \theta_{2,0}\right) \mathrm{d} s}{\hat{\sigma}_{i-1}^{2}} \chi_{\left\{X_{i-1}>\tau^{\prime}\right\}}, \\
& \psi_{3, n}\left(\theta_{2}\right)=\frac{1}{n} \sum_{i=1}^{n} \frac{b_{i-1}^{2}-b_{i-1}^{* 2}}{\hat{\sigma}_{i-1}^{2}} \chi_{\left\{X_{i-1}>\tau^{\prime}\right\}}, \\
& R_{n}\left(\theta_{2}\right)=-\frac{1}{n h_{n}} \sum_{i=1}^{n}\left\{\frac{\left(\Delta_{i} X-b_{i-1} h_{n}\right)^{2}}{\hat{\sigma}_{i-1}^{2} h_{n}}-\frac{\left(\Delta_{i} X-b_{i-1}^{*} h_{n}\right)^{2}}{\hat{\sigma}_{i-1}^{2} h_{n}}\right\} \chi_{\left\{X_{i-1}>\tau^{\prime}, X_{i} \leq \tau\right\}} .
\end{aligned}
$$

We first estimate $R_{n}\left(\theta_{2}\right)$.

$$
\begin{aligned}
E\left[\sup _{\theta_{2}}\left|R_{n}\left(\theta_{2}\right)\right|\right] \leq & \frac{1}{n h_{n}} \sum_{i=1}^{n} E\left[\sup _{\theta_{2}}\left|\frac{\left(\Delta_{i} X-b_{i-1} h_{n}\right)^{2}-\left(\Delta_{i} X-b_{i-1}^{*} h_{n}\right)^{2}}{\hat{\sigma}_{i-1}^{2} h_{n}}\right|^{2}\right]^{1 / 2} \\
& \times P\left(X_{i-1}>\tau^{\prime}, X_{i} \leq \tau\right)^{1 / 2} \\
\leq & \frac{1}{h_{n}^{1 / 2}} \times C\left(\frac{h_{n}^{\alpha}}{\tau^{\prime}-\tau}\right)^{k / 2}\left(h_{n}^{1 / 2-\alpha}\right)^{k / 2} \\
= & O\left(h_{n}^{k / 4-\alpha k / 2-1 / 2}\right) \rightarrow 0,
\end{aligned}
$$

where we took $k>2 /(1-2 \alpha)$ in (11). This yields that $\sup _{\theta_{2}}\left|R_{n}\left(\theta_{2}\right)\right|=o_{p}(1)$. Next, $\psi_{2, n}\left(\theta_{2}\right)$ can be rewritten as

$$
\psi_{2, n}\left(\theta_{2}\right)=\psi_{2, n}^{(1)}\left(\theta_{2}\right)+\psi_{2, n}^{(2)}\left(\theta_{2}\right)+\psi_{2, n}^{(3)}\left(\theta_{2}\right),
$$

where

$$
\begin{aligned}
& \psi_{2, n}^{(1)}\left(\theta_{2}\right)=-\frac{2}{n} \sum_{i=1}^{n} \frac{\left(b_{i-1}-b_{i-1}^{*}\right) b_{i-1}^{*}}{\sigma_{i-1}^{* 2}} \chi_{\left\{X_{i-1}>\tau^{\prime}\right\}}, \\
& \psi_{2, n}^{(2)}\left(\theta_{2}\right)=-\frac{2}{n h_{n}} \sum_{i=1}^{n} \frac{\left(b_{i-1}-b_{i-1}^{*}\right) \int_{t_{i-1}}^{t_{i}}\left\{b\left(X(s), \theta_{2,0}\right)-b_{i-1}^{*}\right\} \mathrm{d} s}{\sigma_{i-1}^{* 2}} \chi_{\left\{X_{i-1}>\tau^{\prime}\right\}}, \\
& \psi_{2, n}^{(3)}\left(\theta_{2}\right)=-\frac{2}{n h_{n}} \sum_{i=1}^{n}\left(b_{i-1}-b_{i-1}^{*}\right) \int_{t_{i-1}}^{t_{i}} b\left(X(s), \theta_{2,0}\right) \mathrm{d} s\left(\frac{1}{\hat{\sigma}_{i-1}^{2}}-\frac{1}{\sigma_{i-1}^{* 2}}\right) \chi_{\left\{X_{i-1}>\tau^{\prime}\right\}} .
\end{aligned}
$$

By noting that for $p>1$ and $K>0$,

$$
\begin{aligned}
& \left\|\int_{t_{i-1}}^{t_{i}}\left\{b\left(X(s), \theta_{2,0}\right)-b_{i-1}^{*}\right\} \mathrm{d} s\right\|_{p} \leq C h_{n}^{3 / 2}, \\
& \left|\frac{1}{\hat{\sigma}_{i-1}^{2}}-\frac{1}{\sigma_{i-1}^{* 2}}\right| \leq C\left|\sigma_{i-1}^{* 2}-\hat{\sigma}_{i-1}^{2}\right| \leq\left|\hat{\theta}_{1, n}-\theta_{1,0}\right| K\left(1+\left|X_{i-1}\right|\right)^{K},
\end{aligned}
$$

one has that for $1<p, q<\infty$ with $1 / p+1 / q=1$,

$$
\begin{aligned}
E\left[\sup _{\theta_{2}}\left|\psi_{2, n}^{(2)}\left(\theta_{2}\right)\right|\right] & \leq \frac{1}{n h_{n}} \sum_{i=1}^{n}\left\|\sup _{\theta_{2}}\left|\frac{b_{i-1}-b_{i-1}^{*}}{\sigma_{i-1}^{* 2}}\right|\right\|_{p} \\
& \times\left\|\int_{t_{i-1}}^{t_{i}}\left\{b\left(X(s), \theta_{2,0}\right)-b_{i-1}^{*}\right\} \mathrm{d} s\right\|_{q} \\
\leq & \frac{C}{n h_{n}} n h_{n}^{3 / 2} \rightarrow 0,
\end{aligned}
$$


and

$$
\begin{aligned}
\sup _{\theta_{2}}\left|\psi_{2, n}^{(3)}\left(\theta_{2}\right)\right| \leq & \left|\hat{\theta}_{1, n}-\theta_{1,0}\right| \frac{K}{n h_{n}} \sum_{i=1}^{n} \sup _{\theta_{2}}\left|b_{i-1}-b_{i-1}^{*}\right| \\
& \times\left|\int_{t_{i-1}}^{t_{i}} b\left(X(s), \theta_{2,0}\right) \mathrm{d} s\right|\left(1+\left|X_{t_{i-1}}\right|\right)^{K} \\
= & o_{p}(1) \times O_{p}(1)=o_{p}(1) .
\end{aligned}
$$

As in the proof of the uniform convergence of (9),

$$
\sup _{\theta_{2}}\left|\psi_{2, n}^{(1)}\left(\theta_{2}\right)+2 \int \frac{\left(b\left(x, \theta_{2}\right)-b\left(x, \theta_{2,0}\right)\right) b\left(x, \theta_{2,0}\right)}{\sigma^{2}\left(x, \theta_{1,0}\right)} \chi_{\{x>\tau\}} v_{\theta_{0}}(\mathrm{~d} x)\right|=o_{p}(1) .
$$

Furthermore, since one estimates

$$
\begin{aligned}
& \sup _{\theta_{2}}\left|\psi_{3, n}\left(\theta_{2}\right)-\frac{1}{n} \sum_{i=1}^{n} \frac{b_{i-1}^{2}-b_{i-1}^{* 2}}{\sigma_{i-1}^{* 2}} \chi_{\left\{X_{i-1}>\tau^{\prime}\right\}}\right| \\
& \leq\left|\hat{\theta}_{1, n}-\theta_{1,0}\right| \frac{K}{n} \sum_{i=1}^{n} \sup _{\theta_{2}}\left|b_{i-1}^{2}-b_{i-1}^{* 2}\right|\left(1+\left|X_{t_{i-1}}\right|\right)^{K} \\
& \quad=o_{p}(1) \times O_{p}(1)=o_{p}(1),
\end{aligned}
$$

we obtain

$$
\sup _{\theta_{2}}\left|\psi_{3, n}\left(\theta_{2}\right)-\int \frac{b\left(x, \theta_{2}\right)^{2}-b\left(x, \theta_{2,0}\right)^{2}}{\sigma^{2}\left(x, \theta_{1,0}\right)} \chi_{\{x>\tau\}} v_{\theta_{0}}(\mathrm{~d} x)\right|=o_{p}(1) .
$$

Therefore, we see that

$$
\sup _{\theta_{2}}\left|\psi_{2, n}\left(\theta_{2}\right)+\psi_{3, n}\left(\theta_{2}\right)-L\left(\theta_{2}\right)\right|=o_{p}(1)
$$

To estimate $\psi_{1}\left(\theta_{1}\right)$, we consider the following process

$$
M_{n}(\theta)=\int_{0}^{n h_{n}} \sum_{i=1}^{n} \frac{\left(b_{i-1}-b_{i-1}^{*}\right) \sigma\left(X(s), \theta_{1,0}\right)}{n h_{n} \sigma_{i-1}^{2}} \mathbf{1}_{i}(s) \mathrm{d} W_{s},
$$

where $\mathbf{1}_{i}(s)=\chi_{\left\{X_{i-1}>\tau^{\prime}\right\}} \chi_{\left\{t_{i-1} \leq s \leq t_{i}\right\}}$. We will prove the following: there exists a constant $\beta>2$ such that for any $\theta$ and $\theta^{\prime}$,

$$
\begin{aligned}
& M_{n}(\theta) \stackrel{p}{\rightarrow} 0, \\
& E\left|M_{n}(\theta)\right|^{\beta} \leq C, \\
& \mathrm{E}\left|M_{n}(\theta)-M_{n}\left(\theta^{\prime}\right)\right|^{\beta} \leq C\left|\theta-\theta^{\prime}\right|^{\beta},
\end{aligned}
$$

where $C$ is a constant independent of $\theta, \theta^{\prime}$ and $n$. If (35)-(37) are satisfied, by Theorem 20 in the Appendix of [8] or Lemma 3.1 of [15], we can show that $\sup _{\theta}\left|M_{n}(\theta)\right| \stackrel{p}{\rightarrow} 0$. In fact, (36) and (37) ensure that the family of distributions of $\left\{M_{n}(\cdot)\right\}$ on $C(\Theta)$ with sup-norm is tight. Hence, if (35)-(37) are shown, one can prove that

$$
\sup _{\theta_{2}}\left|\psi_{1, n}\left(\theta_{2}\right)\right|=2 \sup _{\theta_{2}}\left|M_{n}\left(\hat{\theta}_{1, n}, \theta_{2}\right)\right| \leq 2 \sup _{\theta}\left|M_{n}(\theta)\right| \stackrel{p}{\rightarrow} 0 .
$$


The proof of (37) is as follows. Let us define

$$
\begin{aligned}
f_{i-1}\left(\theta, \theta^{\prime}\right) & =\frac{b_{i-1}\left(\theta_{2}\right)-b_{i-1}^{*}}{\sigma_{i-1}^{2}\left(\theta_{1}\right)}-\frac{b_{i-1}\left(\theta_{2}^{\prime}\right)-b_{i-1}^{*}}{\sigma_{i-1}^{2}\left(\theta_{1}^{\prime}\right)} \\
& =\frac{b_{i-1}\left(\theta_{2}\right)-b_{i-1}\left(\theta_{2}^{\prime}\right)}{\sigma_{i-1}^{2}\left(\theta_{1}^{\prime}\right)}+\left(b_{i-1}\left(\theta_{2}\right)-b_{i-1}^{*}\right)\left(\frac{1}{\sigma_{i-1}^{2}\left(\theta_{1}\right)}-\frac{1}{\sigma_{i-1}^{2}\left(\theta_{1}^{\prime}\right)}\right) .
\end{aligned}
$$

By the Burkholder-Davis-Gundy inequality and the Jensen inequality,

$$
\begin{aligned}
E & \left|M_{n}(\theta)-M_{n}\left(\theta^{\prime}\right)\right|^{\beta} \\
& =\frac{1}{\left(n h_{n}\right)^{\beta}} \mathrm{E}\left|\int_{0}^{n h_{n}} \sum_{i=1}^{n} f_{i-1}\left(\theta, \theta^{\prime}\right) \sigma\left(X(s), \theta_{1,0}\right) \mathbf{1}_{i}(s) \mathrm{d} W_{s}\right|^{\beta} \\
& \leq \frac{C_{\beta}}{\left(n h_{n}\right)^{\beta}} \mathrm{E}\left(\sum_{i=1}^{n} \int_{0}^{n h_{n}}\left(f_{i-1}\left(\theta, \theta^{\prime}\right) \sigma\left(X(s), \theta_{1,0}\right)\right)^{2} \mathbf{1}_{i}(s) \mathrm{d} s\right)^{\frac{\beta}{2}} \\
& \leq \frac{C_{\beta}}{\left(n h_{n}\right)^{\beta}} n^{\beta / 2-1} \sum_{i=1}^{n} \mathrm{E}\left(\int_{t_{i-1}}^{t_{i}}\left(f_{i-1}\left(\theta, \theta^{\prime}\right) \sigma\left(X(s), \theta_{1,0}\right)\right)^{2} \mathrm{~d} s\right)^{\frac{\beta}{2}} \\
& \leq \frac{C_{\beta}}{\left(n h_{n}\right)^{\beta}}\left(n h_{n}\right)^{\beta / 2-1} \sum_{i=1}^{n} \mathrm{E}\left(\int_{t_{i-1}}^{t_{i}}\left|f_{i-1}\left(\theta, \theta^{\prime}\right) \sigma\left(X(s), \theta_{1,0}\right)\right|^{\beta} \mathrm{d} s\right) .
\end{aligned}
$$

Moreover, it follows from A5 to A6,

$$
\left|f_{i-1}\left(\theta, \theta^{\prime}\right)\right|^{\beta} \leq K\left(1+\left|X_{i-1}\right|\right)^{K}\left|\theta-\theta^{\prime}\right|^{\beta},
$$

which completes the proof of (37). In a similar way, we can show (36). For the proof of (35), we set $g_{i}=\left(b_{i}-b_{i}^{*}\right) / \sigma_{i}^{2}$ and

$$
\mathrm{E}\left(M_{n}(\theta)\right)^{2} \leq \frac{1}{n^{2} h_{n}^{2}} \sum_{i=1}^{n} \mathrm{E}\left\{\int_{t_{i-1}}^{t_{i}} g_{i-1}^{2} \sigma^{2}\left(X(s), \theta_{1,0}\right) \mathrm{d} s\right\} \rightarrow 0,
$$

which completes the proof of (35). Thus, we have (38) and this completes the proof of (34). Finally, note that for any $\epsilon>0, \inf _{\theta_{2}:\left|\theta_{2}-\theta_{2,0}\right| \geq \epsilon} L\left(\theta_{2}\right)>0$ because $L$ attains its minimum only at $\theta_{2,0}$. As in the proof of Theorem 3.1, we can show the consistency of $\hat{\theta}_{2, n}$. This completes the proof.

Proof of Theorem 3.3. First, we study the asymptotic normality of the score function. Let

$$
\mathcal{L}_{n}=\left(\begin{array}{l}
-\frac{1}{\sqrt{n}} \partial_{\theta_{1}} g_{n}\left(\theta_{1,0}\right) \\
-\frac{1}{\sqrt{n h_{n}}} \partial_{\theta_{2}} \ell_{n}\left(\hat{\theta}_{1, n}, \theta_{2,0}\right)
\end{array}\right), \quad \overline{\mathcal{L}}_{n}=\left(\begin{array}{c}
-\frac{1}{\sqrt{n}} \partial_{\theta_{1}} \bar{g}_{n}\left(\theta_{1,0}\right) \\
-\frac{1}{\sqrt{n h_{n}}} \partial_{\theta_{2}} \bar{\ell}_{n}\left(\theta_{0}\right)
\end{array}\right),
$$

where

$$
\begin{aligned}
& \bar{g}_{n}\left(\theta_{1}\right)=\sum_{i=1}^{n} g\left(i, i-1 ; \theta_{1}\right) \chi_{\left\{X_{i-1}>\tau^{\prime}\right\}}, \\
& \bar{\ell}_{n}(\theta)=\sum_{i=1}^{n} \ell(i, i-1 ; \theta) \chi_{\left\{X_{i-1}>\tau^{\prime}\right\}} .
\end{aligned}
$$


In order to show that $\mathcal{L}_{n}-\overline{\mathcal{L}}_{n}=o_{p}(1)$, it is sufficient to show that

$$
\begin{aligned}
A_{n} & :=\frac{1}{\sqrt{n}}\left(\partial_{\theta_{1}} g_{n}\left(\theta_{1,0}\right)-\partial_{\theta_{1}} \bar{g}_{n}\left(\theta_{1,0}\right)\right)=o_{p}(1), \\
B_{n} & :=\frac{1}{\sqrt{n h_{n}}}\left(\partial_{\theta_{2}} \ell_{n}\left(\hat{\theta}_{1, n}, \theta_{2,0}\right)-\partial_{\theta_{2}} \bar{\ell}_{n}\left(\theta_{0}\right)\right)=o_{p}(1) .
\end{aligned}
$$

For the proof of (39), one estimates

$$
\begin{aligned}
E\left|A_{n}\right| & \leq \frac{1}{\sqrt{n}} \sum_{i=1}^{n} E\left|\partial_{\theta_{1}} g\left(i-1, i ; \theta_{1,0}\right) \chi_{\left\{X_{i-1}>\tau^{\prime}, X_{i} \leq \tau\right\}}\right| \\
& \leq \frac{C}{\sqrt{n}} \sum_{i=1}^{n}\left\|\frac{\partial_{\theta_{1}} \sigma_{i-1}^{*}}{\sigma_{i-1}^{*}}\left(1-\frac{\left(\Delta_{i} X\right)^{2}}{h_{n} \sigma_{i-1}^{* 2}}\right)\right\|_{2} \times O\left(h_{n}^{(1 / 4-\alpha / 2) k}\right) \\
& \leq C \sqrt{n} h_{n} \times O\left(h_{n}^{(1 / 4-\alpha / 2) k-1}\right) \rightarrow 0,
\end{aligned}
$$

where we took $k>4 /(1-2 \alpha)$ in (11). For the proof of (40), one has that for $\epsilon>0$,

$$
\begin{aligned}
\left|B_{n}\right| \chi_{\left\{\left|\hat{\theta}_{1, n}-\theta_{1,0}\right|<\epsilon\right\}} \leq & \frac{1}{\sqrt{n h_{n}}} \sum_{i=1}^{n} \sup _{\theta_{1}}\left|\partial_{\theta_{1}} \partial_{\theta_{2}} \ell\left(i, i-1 ; \theta_{1}, \theta_{2,0}\right)\right|\left|\hat{\theta}_{1, n}-\theta_{1,0}\right| \\
& +\frac{1}{\sqrt{n h_{n}}} \sum_{i=1}^{n}\left|\partial_{\theta_{2}} \ell\left(i, i-1 ; \theta_{0}\right) \chi_{\left\{X_{i-1}>\tau^{\prime}, X_{i} \leq \tau\right\}}\right| .
\end{aligned}
$$

As in the proof of (39), $\frac{1}{\sqrt{n h_{n}}} \sum_{i=1}^{n}\left|\partial_{\theta_{2}} \ell\left(i, i-1 ; \theta_{0}\right) \chi_{\left\{X_{i-1}>\tau^{\prime}, X_{i} \leq \tau\right\}}\right|=o_{p}(1)$. Next, letting $f_{i-1}\left(\theta_{1}\right)=\frac{\partial_{\theta_{2}} b_{i-1}^{*} \partial_{\theta_{1}} \sigma_{i-1}}{\sigma_{i-1}^{3}}$, we estimate that for $l \geq 1$

$$
\begin{aligned}
E \mid & \left.\frac{1}{\sqrt{n h_{n}}} \sum_{i=1}^{n} \sup _{\theta_{1}}\left|\partial_{\theta_{1}} \partial_{\theta_{2}} \ell\left(i, i-1 ; \theta_{1}, \theta_{2,0}\right)\right|\right|^{2 l} \\
\leq & \frac{C}{\left(n h_{n}\right)^{l}} E\left[\sum_{i=1}^{n} \int_{t_{i-1}}^{t_{i}} \sup _{\theta_{1}}\left|f_{i-1}\left(\theta_{1}\right)\right| \sigma\left(X(s), \theta_{1,0}\right) \mathrm{d} W_{s}\right]^{2 l} \\
& +\frac{C}{\left(n h_{n}\right)^{l}} E\left[\sum_{i=1}^{n} \int_{t_{i-1}}^{t_{i}} \sup _{\theta_{1}}\left|f_{i-1}\left(\theta_{1}\right)\right|\left(b\left(X(s), \theta_{2,0}\right)-b_{i-1}^{*}\right) \mathrm{d} s\right]^{2 l} \\
\leq & \frac{C}{\left(n h_{n}\right)^{l}}\left(n h_{n}\right)^{l-1} \sum_{i=1}^{n} E\left[\int_{t_{i-1}}^{t_{i}} \sup _{\theta_{1}}\left|f_{i-1}\left(\theta_{1}\right)\right|^{2 l} \sigma^{2 l}\left(X(s), \theta_{1,0}\right) \mathrm{d} s\right] \\
& +\frac{C}{\left(n h_{n}\right)^{l}}\left(n h_{n}\right)^{2 l-1} \sum_{i=1}^{n} E\left[\int_{t_{i-1}}^{t_{i}} \sup _{\theta_{1}}\left|f_{i-1}\left(\theta_{1}\right)\right|^{2 l}\left(b\left(X(s), \theta_{2,0}\right)-b_{i-1}^{*}\right)^{2 l} \mathrm{~d} s\right] \\
= & O(1) .
\end{aligned}
$$

Consequently, one has that $\left|B_{n}\right|=o_{p}(1)$.

Next, we will prove that

$$
\overline{\mathcal{L}}_{n} \stackrel{d}{\rightarrow} N(0,4 \Sigma) .
$$


Let

$$
\begin{aligned}
& \xi_{i}^{(1)}=\frac{1}{\sqrt{n}} \partial_{\theta_{1}} \ell\left(i, i-1 ; \theta_{1,0}\right) \chi_{\left\{X_{i-1}>\tau^{\prime}\right\}}=\frac{2}{\sqrt{n}} \frac{\partial_{\theta_{1}} \sigma_{i-1}^{*}}{\sigma_{i-1}^{*}}\left(1-\frac{\left(\Delta_{i} X\right)^{2}}{h_{n} \sigma_{i-1}^{* 2}}\right) \chi_{\left\{X_{i-1}>\tau^{\prime}\right\}}, \\
& \xi_{i}^{(2)}=\frac{1}{\sqrt{n h_{n}}} \partial_{\theta_{2}} \ell\left(i, i-1 ; \theta_{0}\right) \chi_{\left\{X_{i-1}>\tau^{\prime}\right\}} \\
& =-\frac{2}{\sqrt{n h_{n}}}\left\{\partial_{\theta_{2}} b_{i-1}^{*} \frac{\Delta_{i} X-b_{i-1}^{*} h_{n}}{\sigma_{i-1}^{* 2}}\right\} \chi_{\left\{X_{i-1}>\tau^{\prime}\right\}} \text {, } \\
& I\left(\theta_{0}\right)=\left(\begin{array}{cc}
I^{(1,1)}\left(\theta_{1,0}\right) & 0 \\
0 & I^{(2,2)}\left(\theta_{0}\right)
\end{array}\right):=4 \Sigma .
\end{aligned}
$$

In order to obtain (41), by the combination of Theorems 3.2 and 3.4 of [5], it is enough to prove the following convergences.

$$
\begin{aligned}
& \sum_{i=1}^{n} \mathrm{E}_{\theta_{0}}\left\{\xi_{i}^{(m)} \mid \mathcal{F}_{i-1}\right\} \stackrel{p}{\rightarrow} 0, \quad m=1,2, \\
& \sum_{i=1}^{n}\left|\mathrm{E}_{\theta_{0}}\left\{\xi_{i}^{(m)} \mid \mathcal{F}_{i-1}\right\}\right|^{2} \stackrel{p}{\rightarrow} 0, \quad m=1,2, \\
& \sum_{i=1}^{n} \mathrm{E}_{\theta_{0}}\left\{\left(\xi_{i}^{(m)}\right)^{2} \mid \mathcal{F}_{i-1}\right\} \stackrel{p}{\rightarrow} I^{(m, m)}, \quad m=1,2, \\
& \sum_{i=1}^{n} \mathrm{E}_{\theta_{0}}\left\{\xi_{i}^{(1)} \xi_{i}^{(2)} \mid \mathcal{F}_{i-1}\right\} \stackrel{p}{\rightarrow} 0, \\
& \sum_{i=1}^{n} \mathrm{E}_{\theta_{0}}\left\{\left(\xi_{i}^{(m)}\right)^{4} \mid \mathcal{F}_{i-1}\right\} \stackrel{p}{\rightarrow} 0, \quad m=1,2 .
\end{aligned}
$$

For the proof of (42), by using the Itô-Taylor expansion and (32), one has

$$
\sum_{i=1}^{n} \mathrm{E}_{\theta_{0}}\left\{\xi_{i}^{(1)} \mid \mathcal{F}_{i-1}\right\}=\sqrt{n h_{n}^{2}} \cdot \frac{1}{n} \sum_{i=1}^{n} R\left(1, X_{i-1}\right) \stackrel{p}{\rightarrow} 0 .
$$

Moreover, since

$$
\mathrm{E}_{\theta_{0}}\left(X_{i}-X_{i-1} \mid \mathcal{F}_{i-1}\right)=h_{n} b_{i-1}^{*}+R\left(h_{n}^{2}, X_{i-1}\right),
$$

we have

$$
\sum_{i=1}^{n} \mathrm{E}_{\theta_{0}}\left(\xi_{i}^{(2)} \mid \mathcal{F}_{i-1}\right)=\frac{-2 \sqrt{n h_{n}^{3}}}{n} \sum_{i=1}^{n} R\left(1, X_{i-1}\right) \stackrel{p}{\rightarrow} 0 .
$$

This completes the proof of (42). In a similar way,

$$
\begin{aligned}
& \sum_{i=1}^{n}\left|\mathrm{E}_{\theta_{0}}\left\{\xi_{i}^{(1)} \mid \mathcal{F}_{i-1}\right\}\right|^{2}=\frac{h_{n}^{2}}{n} \sum_{i=1}^{n} R\left(1, X_{i-1}\right) \stackrel{p}{\rightarrow} 0, \\
& \sum_{i=1}^{n}\left|\mathrm{E}_{\theta_{0}}\left(\xi_{i}^{(2)} \mid \mathcal{F}_{i-1}\right)\right|^{2}=\frac{h_{n}^{3}}{n} \sum_{i=1}^{n} R\left(1, X_{i-1}\right) \stackrel{p}{\rightarrow} 0,
\end{aligned}
$$


which complete the proof of (43). For the proof of (44), noting that

$$
\begin{aligned}
E\left\{\left(1-\frac{\left(\Delta_{i} X\right)^{2}}{\sigma_{i-1}^{* 2} h_{n}}\right)^{2} \mid \mathcal{F}_{i-1}\right\}= & 1+\frac{3 h_{n}^{2} \sigma_{i-1}^{* 4}+R\left(h_{n}^{5 / 2}, X_{i-1}\right)}{\sigma_{i-1}^{* 4} h_{n}^{2}} \\
& -2 \frac{h_{n} \sigma_{i-1}^{* 2}+R\left(h_{n}^{2}, X_{i-1}\right)}{\sigma_{i-1}^{* 2} h_{n}} \\
= & 2+\sqrt{h_{n}} R\left(1, X_{i-1}\right)
\end{aligned}
$$

one has

$$
\begin{aligned}
\sum_{i=1}^{n} \mathrm{E}_{\theta_{0}}\left\{\left(\xi_{i}^{(1)}\right)^{2} \mid \mathcal{F}_{i-1}\right\} & =\sum_{i=1}^{n} \frac{4}{n}\left(\frac{\partial_{\theta_{1}} \sigma_{i-1}^{*}}{\sigma_{i-1}^{*}}\right)^{2}\left(2+\sqrt{h_{n}} R\left(1, X_{i-1}\right)\right) \chi_{\left\{X_{i-1}>\tau^{\prime}\right\}} \\
& \stackrel{p}{\rightarrow} I^{(1,1)}\left(\theta_{1,0}\right),
\end{aligned}
$$

which proves (44) for $m=1$. It follows from the Itô-Taylor expansion of $\mathrm{E}_{\theta_{0}}\left\{\left(X_{i}-X_{i-1}-\right.\right.$ $\left.\left.h_{n} b_{i-1}^{*}\right)^{2} \mid \mathcal{F}_{i-1}\right\}$ that

$$
\begin{aligned}
\sum_{i=1}^{n} \mathrm{E}_{\theta_{0}}\left\{\left(\xi_{i}^{(2)}\right)^{2} \mid \mathcal{F}_{i-1}\right\} & =\frac{4}{n h_{n}} \sum_{i=1}^{n} \frac{\left(\partial_{\theta_{2}} b_{i-1}^{*}\right)^{2}}{\sigma_{i-1}^{* 4}}\left(h_{n} \sigma_{i-1}^{* 2}+R\left(h_{n}^{2}, X_{i-1}\right)\right) \chi_{\left\{X_{i-1}>\tau^{\prime}\right\}} \\
& \stackrel{p}{\rightarrow} I^{(2,2)}\left(\theta_{0}\right)
\end{aligned}
$$

and (44) is proved. For the proof of (45), we consider

$$
\xi_{i}^{(1)} \xi_{i}^{(2)}=-\frac{4}{n \sqrt{h_{n}}} \frac{\partial_{\theta_{1}} \sigma_{i-1}^{*} \partial_{\theta_{2}} b_{i-1}^{*}}{\sigma_{i-1}^{* 3}}\left(1-\frac{\left(\Delta_{i} X\right)^{2}}{\sigma_{i-1}^{* 2} h_{n}}\right)\left\{\Delta_{i} X-b_{i-1}^{*} h_{n}\right\} \chi_{\left\{X_{i-1}>\tau^{\prime}\right\}} .
$$

Since

$$
\mathrm{E}_{\theta_{0}}\left\{\left(\Delta_{i} X\right)^{2}\left(\Delta_{i} X-b_{i-1}^{*} h_{n}\right) \mid \mathcal{F}_{i-1}\right\}=R\left(h_{n}^{2}, X_{i-1}\right)
$$

and

$$
\mathrm{E}_{\theta_{0}}\left\{\Delta_{i} X-b_{i-1}^{*} h_{n} \mid \mathcal{F}_{i-1}\right\}=R\left(h_{n}^{2}, X_{i-1}\right),
$$

one has

$$
\begin{aligned}
\sum_{i=1}^{n} \mathrm{E}_{\theta_{0}}\left\{\xi_{i}^{(1)} \xi_{i}^{(2)} \mid \mathcal{F}_{i-1}\right\}= & -\frac{4}{n} \sum_{i=1}^{n} \frac{\partial_{\theta_{1}} \sigma_{i-1}^{*} \partial_{\theta_{2}} b_{i-1}^{*}}{\sigma_{i-1}^{* 3}} \chi_{\left\{X_{i-1}>\tau^{\prime}\right\}} \\
& \times \frac{1}{\sqrt{h_{n}}}\left(h_{n}^{2} R\left(1, X_{i-1}\right)-\frac{h_{n} R\left(1, X_{i-1}\right)}{\sigma_{i-1}^{* 2}}\right) \\
\stackrel{p}{\rightarrow} & 0 .
\end{aligned}
$$

Hence (45) is proved. For the proof of (46), using the estimate that for $p \geq 1$,

$$
\mathrm{E}_{\theta_{0}}\left\{\left(\Delta_{i} X\right)^{2 p} \mid \mathcal{F}_{i-1}\right\}=h_{n}^{p} R\left(1, X_{i-1}\right),
$$


one has

$$
\mathrm{E}_{\theta_{0}}\left\{\sum_{i=1}^{n}\left(\xi_{i}^{(1)}\right)^{4} \mid \mathcal{F}_{i-1}\right\} \leq \frac{C^{\prime}}{n} \frac{1}{n} \sum_{i=1}^{n}\left(\frac{\partial_{\theta_{1}} \sigma_{i-1}^{*}}{\sigma_{i-1}^{*}}\right)^{4} \chi_{\left\{X_{i-1}>\tau^{\prime}\right\}}\left\{1+R\left(1, X_{i-1}\right)\right\} \stackrel{p}{\rightarrow} 0,
$$

which completes the proof of (46) for $m=1$. For the case $m=2$, by using the following estimate

$$
\mathrm{E}_{\theta_{0}}\left\{\left(\Delta_{i} X-b_{i-1}^{*} h_{n}\right)^{4} \mid \mathcal{F}_{i-1}\right\}=h_{n}^{2} R\left(1, X_{i-1}\right),
$$

we have that

$$
\sum_{i=1}^{n} \mathrm{E}_{\theta_{0}}\left\{\left(\xi_{i}^{(2)}\right)^{4} \mid \mathcal{F}_{i-1}\right\} \leq \frac{C^{\prime}}{n} \frac{1}{n} \sum_{i=1}^{n}\left(\frac{\partial_{\theta_{2}} b_{i-1}^{*}}{\sigma_{i-1}^{* 2}}\right)^{4} \chi_{\left\{X_{i-1}>\tau^{\prime}\right\}} R\left(1, X_{i-1}\right) \stackrel{p}{\rightarrow} 0 .
$$

Thus (46) is proved. This completes the proof of (41). It follows from (39)-(41) that

$$
\mathcal{L}_{n} \stackrel{d}{\rightarrow} N(0,4 \Sigma) .
$$

Next we consider asymptotic properties of the observed information. Let

$$
D_{n}(\theta)=\left(\begin{array}{cc}
\frac{1}{n} \partial_{\theta_{1}}^{2} g_{n}\left(\theta_{1}\right) & 0 \\
0 & \frac{1}{n h_{n}} \partial_{\theta_{2}}^{2} \ell_{n}\left(\hat{\theta}_{1, n}, \theta_{2}\right)
\end{array}\right), \quad D(\theta)=\left(\begin{array}{cc}
\overline{\mathcal{G}}\left(\theta_{1}\right) & 0 \\
0 & \overline{\mathcal{L}}\left(\theta_{2}\right)
\end{array}\right),
$$

where

$$
\begin{aligned}
\overline{\mathcal{G}}\left(\theta_{1}\right)= & 2 \int_{\mathbf{R}} \frac{\partial_{\theta_{1}}^{2} \sigma\left(X, \theta_{1}\right)}{\sigma^{3}\left(x, \theta_{1}\right)}\left(\sigma^{2}\left(x, \theta_{1}\right)-\sigma^{2}\left(x, \theta_{1,0}\right)\right) \chi_{\{x>\tau\}} v_{\theta_{0}}(\mathrm{~d} x) \\
& +2 \int_{\mathbf{R}} \frac{\left(3 \sigma^{2}\left(x, \theta_{1,0}\right)-\sigma^{2}\left(x, \theta_{1}\right)\right)\left(\partial_{\theta_{1}} \sigma\left(x, \theta_{1}\right)\right)^{2}}{\sigma^{4}\left(x, \theta_{1}\right)} \chi_{\{x>\tau\}} v_{\theta_{0}}(\mathrm{~d} x), \\
\overline{\mathcal{L}}\left(\theta_{2}\right)= & 2 \int_{\mathbf{R}}\left(\frac{\partial_{\theta_{2}} b\left(x, \theta_{2}\right)}{\sigma\left(x, \theta_{1,0}\right)}\right)^{2} \chi_{\{x>\tau\}} v_{\theta_{0}}(\mathrm{~d} x) \\
& -2 \int_{\mathbf{R}} \frac{\left(b\left(x, \theta_{2,0}\right)-b\left(x, \theta_{2}\right)\right) \partial_{\theta_{2}}^{2} b\left(x, \theta_{2}\right)}{\sigma^{2}\left(x, \theta_{1,0}\right)} \chi_{\{x>\tau\}} v_{\theta_{0}}(\mathrm{~d} x) .
\end{aligned}
$$

In order to prove that

$$
\sup _{\theta}\left|D_{n}(\theta)-D(\theta)\right|=o_{p}(1),
$$

it is sufficient to show that

$$
\begin{aligned}
& \sup _{\theta_{1}}\left|\frac{1}{n} \partial_{\theta_{1}}^{2} g_{n}\left(\theta_{1}\right)-\frac{1}{n} \partial_{\theta_{1}}^{2} \bar{g}_{n}\left(\theta_{1}\right)\right|=o_{p}(1), \\
& \sup _{\theta}\left|\frac{1}{n h_{n}} \partial_{\theta_{2}}^{2} \ell_{n}(\theta)-\frac{1}{n h_{n}} \partial_{\theta_{2}}^{2} \bar{\ell}_{n}(\theta)\right|=o_{p}(1), \\
& \sup _{\theta_{1}}\left|\frac{1}{n} \partial_{\theta_{1}}^{2} \bar{g}_{n}\left(\theta_{1}\right)-\overline{\mathcal{G}}\left(\theta_{1}\right)\right|=o_{p}(1), \\
& \sup _{\theta_{2}}\left|\frac{1}{n h_{n}} \partial_{\theta_{2}}^{2} \bar{\ell}_{n}\left(\hat{\theta}_{1, n}, \theta_{2}\right)-\overline{\mathcal{L}}\left(\theta_{2}\right)\right|=o_{p}(1) .
\end{aligned}
$$


For the proof of (49), as in the proof of the uniform convergence of (10), one has that

$$
\begin{aligned}
& \mathrm{E}\left\{\sup _{\theta_{1}}\left|\frac{1}{n} \sum_{i=1}^{n} \partial_{\theta_{1}}^{2} g\left(i, i-1 ; \theta_{1}\right) \chi_{\left\{X_{i-1}>\tau^{\prime}, X_{i} \leq \tau\right\}}\right|\right\} \\
& \quad \leq \frac{1}{n} \sum_{i=1}^{n}\left\|\sup _{\theta_{1}}\left|\partial_{\theta_{1}}^{2} g\left(i, i-1 ; \theta_{1}\right)\right|\right\|_{2} P\left(X_{i-1}>\tau^{\prime}, X_{i} \leq \tau\right)^{\frac{1}{2}} \rightarrow 0 .
\end{aligned}
$$

For the proof of (50), in a quite similar way as in the proof of (49), one has that

$$
\begin{aligned}
& \mathrm{E}\left\{\sup _{\theta}\left|\frac{1}{n h_{n}} \sum_{i=1}^{n} \partial_{\theta_{1}}^{2} \ell(i, i-1 ; \theta) \chi_{\left\{X_{i-1}>\tau^{\prime}, X_{i} \leq \tau\right\}}\right|\right\} \\
& \quad \leq \frac{1}{n h_{n}} \sum_{i=1}^{n}\left\|\sup _{\theta}\left|\partial_{\theta_{1}}^{2} \ell(i, i-1 ; \theta)\right|\right\|_{2} P\left(X_{i-1}>\tau^{\prime}, X_{i} \leq \tau\right)^{\frac{1}{2}} \\
& \quad \leq C \frac{1}{h_{n}^{1 / 2}} \times\left(\frac{h_{n}^{\alpha}}{\tau^{\prime}-\tau}\right)^{k / 2}\left(h_{n}^{1 / 2-\alpha}\right)^{k / 2} \\
& =O\left(h_{n}^{k / 4-\alpha k / 2-1 / 2}\right) \rightarrow 0,
\end{aligned}
$$

where we took $k>2 /(1-2 \alpha)$ in (11). For the proof of (51), we set

$$
\begin{aligned}
& \eta_{i}\left(\theta_{1}\right)=\frac{1}{n} \partial_{\theta_{1}}^{2} g\left(i, i-1 ; \theta_{1}\right) \chi_{\left\{X_{i-1}>\tau^{\prime}\right\}} \\
&= \frac{2}{n h_{n} \sigma_{i-1}^{4}} \\
& \quad \times\left\{\left(3\left(\Delta_{i} X\right)^{2}-h_{n} \sigma_{i-1}^{2}\right)\left(\partial_{\theta_{1}} \sigma_{i-1}\right)^{2}+\sigma_{i-1}\left(h_{n} \sigma_{i-1}^{2}-\left(\Delta_{i} X\right)^{2}\right) \partial_{\theta_{1}}^{2} \sigma_{i-1}\right\} \chi_{\left\{X_{i-1}>\tau^{\prime}\right\}} .
\end{aligned}
$$

It follows from standard arguments that

$$
\sum_{i=1}^{n} \mathrm{E}_{\theta_{0}}\left\{\eta_{i}\left(\theta_{1}\right) \mid \mathcal{F}_{i-1}\right\} \stackrel{p}{\rightarrow} \overline{\mathcal{G}}\left(\theta_{1}\right), \quad \sum_{i=1}^{n} \mathrm{E}_{\theta_{0}}\left\{\left(\eta_{i}\left(\theta_{1}\right)\right)^{2} \mid \mathcal{F}_{i-1}\right\} \stackrel{p}{\rightarrow} 0
$$

Therefore one has that for each $\theta_{1}$,

$$
\frac{1}{n} \partial_{\theta_{1}}^{2} \bar{g}_{n}\left(\theta_{1}\right) \stackrel{p}{\rightarrow} \overline{\mathcal{G}}\left(\theta_{1}\right)
$$

It is easy to show that $\sup _{n} E\left[\sup _{\theta_{1}}\left|\frac{1}{n} \partial_{\theta_{1}}^{3} \bar{g}_{n}\left(\theta_{1}\right)\right|\right]<\infty$, which completes the proof of (51). For the proof of (52), we set

$$
\frac{1}{n h_{n}} \partial_{\theta_{2}}^{2} \bar{\ell}_{n}\left(i, i-1 ; \hat{\theta}_{1, n}, \theta_{2}\right)=\Xi_{1}\left(\theta_{2}\right)+\Xi_{2}\left(\theta_{2}\right)+\Xi_{3}\left(\theta_{2}\right)
$$

where

$$
\begin{aligned}
& \Xi_{1}\left(\theta_{2}\right)=\frac{2}{n} \sum_{i=1}^{n}\left\{\left(\frac{\partial_{\theta_{2}} b_{i-1}}{\hat{\sigma}_{i-1}}\right)^{2}-\frac{\left(b_{i-1}^{*}-b_{i-1}\right) \partial_{\theta_{2}}^{2} b_{i-1}}{\hat{\sigma}_{i-1}^{2}}\right\} \chi_{\left\{X_{i-1}>\tau^{\prime}\right\}}, \\
& \Xi_{2}\left(\theta_{2}\right)=-\frac{2}{n h_{n}} \sum_{i=1}^{n} \frac{\partial_{\theta_{2}}^{2} b_{i-1} \int_{t_{i-1}}^{t_{i}}\left\{b\left(X(s), \theta_{2,0}\right)-b_{i-1}^{*}\right\} \mathrm{d} s}{\hat{\sigma}_{i-1}^{2}} \chi_{\left\{X_{i-1}>\tau^{\prime}\right\}}
\end{aligned}
$$




$$
\Xi_{3}\left(\theta_{2}\right)=-\frac{2}{n h_{n}} \sum_{i=1}^{n} \frac{\partial_{\theta_{2}}^{2} b_{i-1} \int_{t_{i-1}}^{t_{i}} \sigma\left(X(s), \theta_{1,0}\right) \mathrm{d} W_{s}}{\hat{\sigma}_{i-1}^{2}} \chi_{\left\{X_{i-1}>\tau^{\prime}\right\}}
$$

In a quite similar way as in the proof of (34), one has that

$$
\sup _{\theta_{2}}\left|\Xi_{1}\left(\theta_{2}\right)-\overline{\mathcal{L}}\left(\theta_{2}\right)\right|=o_{p}(1), \quad \sup _{\theta_{2}}\left|\Xi_{2}\left(\theta_{2}\right)\right|=o_{p}(1), \quad \sup _{\theta_{2}}\left|\Xi_{3}\left(\theta_{2}\right)\right|=o_{p}(1) .
$$

This completes the proof of (52). Thus, (48) is proved.

By the Taylor expansion, $\int_{0}^{1} D_{n}\left(\theta_{0}+u\left(\hat{\theta}_{n}-\theta_{0}\right)\right) \mathrm{d} u S_{n}=\mathcal{L}_{n}$ on an event with probability tending to one, where $S_{n}=\left(\begin{array}{c}\sqrt{n}\left(\hat{\theta}_{1, n}-\theta_{1,0}\right) \\ \sqrt{n h_{n}}\left(\hat{\theta}_{2, n}-\theta_{2,0}\right)\end{array}\right)$. It follows from (47) that

$$
\mathcal{L}_{n} \stackrel{d}{\rightarrow} N(0,4 \Sigma) .
$$

By (48) and the continuity of $D(\theta)$ with respect to $\theta$, one has

$$
\begin{aligned}
& D_{n}\left(\theta_{0}\right) \stackrel{p}{\rightarrow} 2 \Sigma, \\
& \sup _{|\theta| \leq \epsilon_{n}}\left|D_{n}\left(\theta_{0}+\theta\right)-D_{n}\left(\theta_{0}\right)\right|=o_{p}(1)
\end{aligned}
$$

for any sequence $\epsilon_{n}$ of positive numbers tending to zero. By using (53)-(55), it is easy to obtain the desired result. This completes the proof.

\section{Acknowledgments}

The authors wish to thank the referee, the associate editor and the editor for their valuable comments. This work was done during the period of stay of the first author at the ISM (Institute of Statistical Mathematics) and the University of Tokyo. The ISM is acknowledged with thanks. The work has been supported by the JSPS (Japan Society for the Promotion of Science) Program FY2006, grant ID No. S06174. Masayuki Uchida's research was partially supported by Grantin-Aid for Scientific Research (C) No. 19540137 from the JSPS, and by Cooperative Research Program of the ISM. Nakahiro Yoshida's research was in part supported by Grant-in-Aid for Scientific Research (B) No. 19340021 from the JSPS, and by Cooperative Research Program of the ISM.

\section{References}

[1] P. Fearnhead, O. Papaspiliopoulos, G.O. Roberts, Particle filters for partially observed diffusions, J. Roy. Statist. Soc. Ser. B 70 (2008) 755-777.

[2] D. Florens-Zmirou, Approximate discrete time schemes for statistics of diffusion processes, Statistics 20 (1989) 547-557.

[3] V. Genon-Catalot, J. Jacod, On the estimation of the diffusion coefficient for multidimensional diffusion processes, Ann. Inst. Henri Poincaré Probab. Statist. 29 (1993) 119-151.

[4] P.E. Greenwood, L.M. Ward, W. Wefelmeyer, Statistical analysis of stochastic resonance in a simple setting, Phys. Rev. E 60 (2000) 4687-4695.

[5] P. Hall, C. Heyde, Martingale Limit Theory and its Applications, Academic Press, New York, 1980.

[6] S.M. Iacus, Statistical analysis of stochastic resonance with ergodic diffusion noise, Stochast. Stochast. Rep. 73 (2002) 271-285.

[7] S.M. Iacus, I. Negri, Estimating unobservable signal with Markovian noise induction, Statist. Methods Appl. 12 (2003) 153-167.

[8] I.A. Ibragimov, R.Z. Has'minskii, Statistical Estimation, Springer-Verlag, New York, 1981.

[9] M. Kessler, Estimation of an ergodic diffusion from discrete observations, Scand. J. Statist. 24 (1997) $211-229$. 
[10] J.R. Movellan, P. Mineiro, A Monte Carlo EM approach for partially observable diffusion processes: Theory and applications to neural networks, Neural Computation 14 (2002) 1507-1544.

[11] B.L.S. Prakasa Rao, Asymptotic theory for nonlinear least squares estimator for diffusion processes, Math. Operationsforsch. Statist. Ser. Statist. 14 (1983) 195-209.

[12] B.L.S. Prakasa Rao, Statistical Inference from Sampled Data for Stochastic Processes, in: Contemp. Math., vol. 80, Amer. Math. Soc., Providence, RI, 1988, pp. 249-284.

[13] G.O. Roberts, O. Stramer, Likelihood based inference for observed and partially observed diffusions, Biometrika 88 (2001) 603-621.

[14] J.L. Starck, H. Aussel, D. Elbaz, D. Fadda, C. Cesarsky, Faint source detection in ISOCAM images, Astron. Astrophys. Suppl. Ser. 138 (1999) 365-379.

[15] N. Yoshida, Asymptotic behavior of $M$-estimator and related random field for diffusion process, Ann. Inst. Statist. Math. 42 (1990) 221-251.

[16] N. Yoshida, Estimation for diffusion processes from discrete observations, J. Multivar. Anal. 41 (1992) $220-242$.

[17] N. Yoshida, Polynomial type large deviation inequalities and quasi-likelihood analysis for stochastic differential equations, 2005. Ann. Inst. Statist. Math. (in press).

[18] Y. Zeng, A partially observed model for micromovement of asset prices with Bayes estimation via filtering, Math. Finance 13 (2003) 411-444. 